\title{
Self-Similar Exponential Approximants
}

\author{
V. I. Yukalov ${ }^{1,2}$ and S. Gluzman ${ }^{3}$ \\ ${ }^{1}$ Centre for Interdisciplinary Studies in Chemical Physics \\ University of Western Ontario, London, Ontario N6A 3K7, Canada \\ ${ }^{2}$ Bogolubov Laboratory of Theoretical Physics \\ Joint Institute for Nuclear Research, Dubna 141980, Russia \\ ${ }^{3}$ International Centre of Condensed Matter Physics \\ University of Brasilia, CP 04513, Brasilia, DF 70919-970, Brazil
}

\begin{abstract}
An approach is suggested defining effective sums of divergent series in the form of self-similar exponential approximants. The procedure of constructing these approximants from divergent series with arbitrary noninteger powers is developed. The basis of this construction is the self-similar approximation theory. Control functions governing the convergence of exponentially renormalized series are defined from stability and fixed-point conditions and from additional asymptotic conditions when the latter are available. The stability of the calculational procedure is checked by analyzing cascade multipliers. A number of physical examples for different statistical systems illustrate the generality and high accuracy of the approach.
\end{abstract}

02.30Lt, 05.70Jk, 64.60Ak, $64.60 \mathrm{Fr}$

\section{INTRODUCTION}

Summation of divergent series is the problem of great importance in theoretical physics, applied mathematics and engineering. This is because realistic problems are usually solved by means of some calculational algorithm often resulting in divergent sequences of approximations. Assigning a finite value to the limit of a divergent sequence is called renormalization or summation technique. The most widely used such technique is Padé summation [1]. However, the latter has several shortcomings. First of all, to reach a reasonable accuracy of Padé approximants, one needs to know at least in the magnitude of ten to twenty terms of a perturbation series. Unfortunately, so many terms are often not available because of the complexity of a considered problem. Second, Padé approximants are defined for the series of integer powers. But in many cases asymptotic series arise having noninteger powers. Third, there are quite simple examples [2] that are not Padé summable even for a sufficiently small variable. Moreover, the standard Padé approximants do not converge at infinity, since infinity is an essential singularity [1]. The latter deficiency can be sometimes treated with the help of two-point Padé approximants [3]. But these can only be constructed when there are two perturbation expansions in the vicinity of two points, so that such expansions have compatible variables, which often is not the case, especially when one of or both these expansions contain noninteger powers [4]. For instance, only rational powers can be described at infinity [3]. This is because a Padé approximant is a ratio of two polynomials, say, of $m$ and $n$ order. Therefore, when the variable tends to infinity, the asymptotic behaviour of this Padé approximant is of power law with the power $m / n$, which is a rational number. Fifth, in many physical problems the quantities of interest exhibit at infinity exponential behaviour, which in principle cannot be described by Padé approximants. One more well known difficulty when dealing with Padé approximants is the appearance of spurious poles [1]. The last, but not the least, Padé summation is rather a numerical technique providing answers in the form of numbers. Therefore, it is difficult, if possible, to analyze the results when the considered problem contains several parameters to be varied, since for each given set of parameters one has to repeat the whole procedure of constructing a table of Padé approximants and selecting of them one corresponding to a visible saturation of numerical values. Being a numerical technique, Padé summation shares the difficulties of other numerical methods, like nonlinear sequence transformations, and sometimes is less effective than the latter $[5,6]$.

The aim of the present paper is to develop an analytical method, free of the Padé approximation difficulties, for summing divergent series containing any number of terms ( just a few or many) with arbitrary noninteger powers. The method is based on the ideas of the self-similar approximation theory [7-14] in its algebraically invariant formulation [12-14]. The novelty of this paper is in what follows:

(i) The approach is generalized by constructing self-similar exponential approximants from the series with arbitrary powers, integer as well as noninteger. (ii) It is shown how such exponential approximants can be made compatible with additional asymptotic and boundary conditions. (iii) Stability and fixed-point conditions are discussed and 
concrete prescriptions, for defining control functions and for checking the stability of the calculational procedure, are formulated. (iv) It is demonstrated that the approach is applicable in all cases, when either just a few terms of a series are known or when many terms are available. (v) We emphasize that in all the cases, even when a large number of terms of a series are involved, we obtain analytical formulae that are convenient for considering with respect to a change of physical parameters. The possibility of deriving analytical expressions is a characteristic feature of our approach differing it from numerical methods.

Also, we illustrated the generality of the approach by applying it to several interesting physical problems of quite different nature. Some of these problems could be treated by Padé summation or by the standard renormalizationgroup technique. When such treatments have been done, we compare their results with ours. In those cases for which numerical data are available, we estimate the accuracy and show that this accuracy is either comparable or higher than that of other more complicated resummation techniques. We construct as well self-similar exponential approximants for several physical problems for which other summation methods have not been applied because of technical difficulties.

\section{NONINTEGER POWERS}

The main idea [7] of the self-similar approximation theory is to put into correspondence to a sequence of perturbative terms a dynamical system for which the approximation number would play the role of discrete time. Then the transformation from one approximation to another can be represented as the evolution of this dynamical system [7]. The corresponding evolution equation may be formulated as the property of functional self-similarity, which is a necessary condition of fast convergence [8]. A dynamical system with discrete time is called a cascade. A fixed point of a cascade, representing a sequence of approximations, corresponds to an effective limit of this sequence $[7,8]$. To guarantee convergence, the fixed point must be stable and the sufficient conditions of stability can be formulated in terms of multipliers [9-11]. Additional possibilities open if we require that the procedure of finding an effective limit of a sequence is invariant with respect to algebraic transformations [12-14], which permits us to deal not with the initial sequence of approximations but with a sequence of its transforms. General ideas and the mathematical foundation of the self-similar approximation theory have been described in detail in our previous papers [7-14]. Not repeating them here, we begin at once with considering the case when for a sought function $f(x)$ one derives an approximate perturbative expression

$$
p_{k}(x)=\sum_{n=0}^{k} a_{n} x^{\alpha_{n}},
$$

in which $\alpha_{n}$ are arbitrary real numbers, integer or noninteger, positive or negative, but with the sole requirement that they form an ordered sequence $\left\{\alpha_{n}\right\}$, that is, either strictly increasing or strictly decreasing sequence of terms. Following the method of the algebraic self-similar renormalization [12-14], we define the algebraic transform

$$
P_{k}(x, s) \equiv x^{s} p_{k}(x)=\sum_{n=0}^{k} a_{n} x^{s+\alpha_{n}},
$$

where $s$ is real. Then, by means of the equation

$$
P_{0}(x, s)=a_{0} x^{s+\alpha_{0}}=\varphi,
$$

we obtain the expansion function

$$
x(\varphi, s)=\left(\frac{\varphi}{a_{0}}\right)^{1 /\left(s+\alpha_{0}\right)} .
$$

Substituting the latter into Eq. (2), we have

$$
y_{k}(\varphi, s) \equiv P_{k}(x(\varphi, s), s)=\sum_{n=0}^{k} a_{n}\left(\frac{\varphi}{a_{0}}\right)^{\left(s+\alpha_{n}\right) /\left(s+\alpha_{0}\right)} .
$$

The family $\left\{y_{k}\right\}$ of transforms (5) is called $[10,11]$ the approximation cascade, since its trajectory $\left\{y_{k}(\varphi, s) \mid k=\right.$ $0,1,2 \ldots\}$ is bijective to the sequence $\left\{P_{k}(x, s) \mid k=0,1,2 \ldots\right\}$ of approximations (2). A cascade is a dynamical system 
in discrete time $k=0,1,2 \ldots$, whose trajectory points satisfy the semigroup property $y_{k+p}(\varphi, s)=y_{k}\left(y_{p}(\varphi, s), s\right)$. The latter equation is a particular type of functional equations [15] and in dynamical theory it is related to autonomous dynamical systems [16]. The physical meaning of the above semigroup relation can be understood as the property of functional self-similarity [17] with respect to the varying approximation number [7-11]. The self-similarity relation is a necessary condition for the fastest convergence criterion $[8,9]$.

For the approximation cascade $\left\{y_{k}\right\}$, defined by transform (5), the cascade velocity can be written as a finite difference

$$
v_{k}(\varphi, s) \equiv y_{k}(\varphi, s)-y_{k-1}(\varphi, s)=a_{k}\left(\frac{\varphi}{a_{0}}\right)^{\left(s+\alpha_{k}\right) /\left(s+\alpha_{0}\right)} .
$$

This is to be substituted into the evolution integral

$$
\int_{P_{k-1}}^{P_{k}^{*}} \frac{d \varphi}{v_{k}(\varphi, s)}=\tau
$$

in which $P_{k}=P_{k}(x, s)$ and $\tau$ is the minimal time needed for reaching a fixed point $P_{k}^{*}=P_{k}^{*}(x, s, \tau)$. Integral (7) with velocity $(6)$ yields

$$
P_{k}^{*}(x, s, \tau)=\left[P_{k-1}^{-\nu}(x, s)-\frac{\nu a_{k} \tau}{a_{0}^{1+\nu}}\right]^{-1 / \nu},
$$

where

$$
\nu=\nu_{k}(s) \equiv \frac{\alpha_{k}-\alpha_{0}}{s+\alpha_{0}} .
$$

Taking the algebraic transform, inverse to Eq.(2), we find

$$
p_{k}^{*}(x, s, \tau) \equiv x^{-s} P_{k}^{*}(x, s, \tau)=\left[p_{k-1}^{-\nu}(x)-\frac{\nu a_{k} \tau}{a_{0}^{1+\nu}} x^{s \nu}\right]^{-1 / \nu} .
$$

Exponential renormalization $[13,14]$ corresponds to the limit $s \rightarrow \infty$, at which

$$
\lim _{s \rightarrow \infty} \nu_{k}(s)=0, \quad \lim _{s \rightarrow \infty} s \nu_{k}(s)=\alpha_{k}-\alpha_{0} .
$$

Then Eq. (10) gives

$$
\lim _{s \rightarrow \infty} p_{k}^{*}(x, s, \tau)=p_{k-1}(x) \exp \left(\frac{a_{k}}{a_{0}} \tau x^{\alpha_{k}-\alpha_{0}}\right) .
$$

Accomplishing exponential renormalization of all sums appearing in expressions of type (11), we follow the bootstrap procedure [14] according to the scheme

$$
p_{k}(x) \rightarrow p_{k}^{*}(x, s, \tau) \rightarrow F_{k}\left(x, \tau_{1}, \tau_{2}, \ldots, \tau_{k}\right),
$$

with $k \geq 1$.

Let us illustrate explicitly how this exponential bootstrap works. The initial approximation from Eq.(1) is

$$
p_{0}(x)=a_{0} x^{\alpha_{0}} .
$$

If we limit ourselves by the first-order term

$$
p_{1}(x)=p_{0}(x)+a_{1} x^{\alpha_{1}},
$$

then, following the renormalization scheme (12), we get

$$
F_{1}\left(x, \tau_{1}\right)=p_{0}(x) \exp \left(b_{1} x^{\beta_{1}}\right),
$$

where 


$$
b_{1} \equiv \frac{a_{1}}{a_{0}} \tau_{1}, \quad \beta_{1} \equiv \alpha_{1}-\alpha_{0} .
$$

Involving the second order term

$$
p_{2}(x)=p_{1}(x)+a_{2} x^{\alpha_{2}},
$$

we find

$$
F_{2}\left(x, \tau_{1}, \tau_{2}\right)=p_{0}(x) \exp \left(b_{1} x^{\beta_{1}} \exp \left(b_{2} x^{\beta_{2}}\right)\right),
$$

with the notation

$$
b_{2} \equiv \frac{a_{2}}{a_{1}} \tau_{2}, \quad \beta_{2} \equiv \alpha_{2}-\alpha_{1} .
$$

Continuing the same procedure, for the $k$-th order expression (1) we obtain

$$
F_{k}\left(x, \tau_{1}, \tau_{2}, \ldots, \tau_{k}\right)=p_{0}(x) \exp \left(b_{1} x^{\beta_{1}} \exp \left(b_{2} x^{\beta_{2}} \ldots \exp \left(b_{k} x^{\beta_{k}}\right)\right) \ldots\right),
$$

where

$$
b_{k} \equiv \frac{a_{k}}{a_{k-1}} \tau_{k}, \quad \beta_{k} \equiv \alpha_{k}-\alpha_{k-1} .
$$

The quantities $\tau_{n}$, with $n=1,2, \ldots k$, in the renormalized form (18) play the role of control functions [7-14]. In the following section we shall show the ways of defining these control functions. Assume, for a while, that the latter have already been defined giving $\tau_{n}=\tau_{n}(x)$. Substituting these functions into Eq.(18), we come to the self-similar exponential approximant

$$
f_{k}^{*}(x)=F_{k}\left(x, \tau_{1}(x), \tau_{2}(x), \ldots, \tau_{k}(x)\right) .
$$

Note that the constructed approximants (20) are different from the iterated exponentials introduced by Euler [18]; the properties of such iterated exponentials being reviewed, e.g., in Refs. [19,20].

Although in this paper we shall deal with physical problems related to series with powers being real numbers, nothing prevents us from generalizing the whole approach to series with complex powers. Such complex exponents appear in the problems with discrete scale invariance [21,22], which has recently been documented in the models of rupture, earthquake processes, financial crashes, in the fractal geometry of growth processes, and in several random systems [21-24]. Our approach can be straightforwardly applied to series with complex powers. Then, the variable $s$ in the algebraic transform (2) has also to be considered as complex. Therefore, the corresponding control functions $s_{k}(x)$ become complex. However, before passing to the general case of complex powers and, respectively, of complex control functions, we need first to develop the approach for arbitrary real powers and real control functions.

\section{CONTROL FUNCTIONS}

The role of control functions, by their definition [26], is to provide convergence for initially divergent sequences. There are several ways of incorporating control functions into an iterative algorithm and of defining them. One way of introducing these functions is by including them into an initial approximation. The most often used definitions of control functions are through the minimal-difference [26-28] or minimal-sensitivity [29-36] conditions. In some very simple cases, like zero-dimensional and one-dimensional oscillators, when high-order terms of perturbation theory are available explicitly, control functions can be found from the direct observation of convergence of this theory [37-40]. All these variants are particular types of quasifixed-point conditions [7-11].

Another way of introducing control functions is through an algebraic transformation [12-14]. In addition, the minimal time appearing in the evolution integral (7) under each renormalization step plays also the role of a control function. After $k$ steps of the renormalization procedure, the self-similar approximation (20) contains $k$ time-like control functions $\tau_{n}, n=1,2, \ldots k$.

The simplest way of defining these control functions would be to remember that the effective time in integral (7) corresponds to the minimal number of steps needed for reaching a fixed point. When no other restrictions are imposed, the minimal number of steps is, clearly, equal to one. Putting $\tau_{n}=1$ for all $n=1,2, \ldots k$ in Eq.(20) gives

$$
f_{k}^{*}(x)=F_{k}(x, 1,1, \ldots, 1) .
$$


A more elaborate definition of the time-like control functions can be formulated by involving one of the variants of fixed-point conditions. To this end, let us put $\tau_{n}=1$ for all $n=1,2, \ldots k-1$, except the last step for which $\tau_{n}=\tau$ is yet undefined. Consider the sequence $\left\{f_{k}\right\}$ consisting of the terms

$$
f_{k}(x, \tau) \equiv F_{k}(x, 1,1, \ldots, \tau)
$$

Following the standard procedure [10-14], it is possible to construct an approximation cascade with a trajectory bijective to the sequence $\left\{f_{k}\right\}$. For the cascade velocity we may write the finite difference

$$
V_{k}(x, \tau)=f_{k}(x, \tau)-f_{k-1}(x, \tau) .
$$

When approaching a fixed point, the cascade velocity tends to zero. Therefore, the condition to be as close to the fixed point as possible is the minimum of velocity (23). This condition

$$
\min _{\tau}\left|V_{k}(x, \tau)\right|=\left|V_{k}\left(x, \tau_{k}(x)\right)\right|
$$

provides us the definition of the time-control function $\tau_{k}(x)$. Eq.(24) is the general form of the minimal-velocity condition $[12,41]$. In particular, taking into account definition $(23)$, we may have the equation

$$
f_{k}(x, \tau)-f_{k-1}(x, \tau)=0,
$$

whose solution $\tau=\tau_{k}(x)$ is the sought control function. For the exponential approximants (18), Eq. (25) yields the equation

$$
\tau=\exp \left(\frac{a_{k}}{a_{k-1}} \tau x^{\beta_{k}}\right) .
$$

The solution to Eq. (26), that is, $\tau=\tau_{k}(x)$, being substituted into (22), leads us to the self-similar approximation

$$
f_{k}^{*}(x)=f_{k}\left(x, \tau_{k}(x)\right) .
$$

The described scheme of defining control functions is applicable when no additional restrictions are imposed on the behavior of the sought function $f(x)$. It may happen, that, in addition to expansion (1), an asymptotic behavior of $f(x)$, as $x \rightarrow x_{0}$, is known. Then the asymptotic condition

$$
f(x) \simeq f_{0}(x), \quad x \rightarrow x_{0}
$$

plays the role of an imposed restriction. And the constructed self-similar approximations are assumed to satisfy the asymptotic condition (28). In such circumstances, some of control functions are to be chosen so that condition (28) be valid. This can be done in the following way.

Let us renormalize a series $p_{k-1}(x)$ to a self-similar approximation $f_{k-1}^{*}(x)$ with time-like control functions defined according to a scheme described above. Limiting ourselves by such a $(k-1)$-step renormalization, we obtain from Eq.(10)

$$
F_{k}^{*}(x, s, \tau)=\left[\left(f_{k-1}^{*}(x)\right)^{-\nu}-\frac{\nu a_{k} \tau}{a_{0}^{1+\nu}} x^{s \nu}\right]^{-1 / \nu},
$$

with the same notation (9). Now we require that the obtained expression (29) would satisfy the asymptotic condition

$$
F_{k}^{*}(x, s, \tau) \simeq f_{0}(x), \quad x \rightarrow x_{0},
$$

in accordance with (28). The control functions $s=s_{k}(x)$ and $\tau=\tau_{k}(x)$ are to be chosen so that condition (30) be valid. With the so defined control functions, we come to the self-similar approximation

$$
f_{k}^{*}(x)=F_{k}^{*}\left(x, s_{k}(x), \tau_{k}(x)\right)
$$

possessing the desired asymptotic property (28).

To clearly illustrate the latter variant of defining control functions, consider the integral

$$
J(g)=\frac{1}{\sqrt{\pi}} \int_{-\infty}^{\infty} \exp \left(-x^{2}-g x^{4}\right) d x,
$$


which has the meaning of the partition function for the zero-dimensional anharmonic model, where $\mathrm{g}$ is called a coupling parameter. The series for integral (32) are frequently used as a model for strongly divergent perturbation expansions in quantum field theory [25].

The weak-coupling expansion of (32) gives

$$
J(g) \simeq a+b g+\ldots, \quad(g \rightarrow 0),
$$

with $a=1, b=-\frac{3}{4}$. In the strong-coupling limit one has

$$
J(g) \simeq A g^{-1 / 4}+B g^{-3 / 4}+C g^{-5 / 4}+D g^{-7 / 4} \ldots, \quad(g \rightarrow \infty),
$$

where

$$
A=\frac{1.813}{\sqrt{\pi}}, \quad B=-\frac{0.612}{\sqrt{\pi}}, \quad C=\frac{0.227}{\sqrt{\pi}} .
$$

The strong-coupling expansion (34) is of the form of series (1) with the coefficients

$$
a_{0}=A, \quad a_{1}=B, \quad a_{2}=C, \quad a_{3}=D
$$

and with noninteger powers

$$
\alpha_{0}=-\frac{1}{4}, \quad \alpha_{1}=-\frac{3}{4}, \quad \alpha_{2}=-\frac{5}{4}, \quad \alpha_{3}=-\frac{7}{4} .
$$

We shall renormalize expansion (34) subject to the limiting condition

$$
\lim _{g \rightarrow 0} J(g)=a,
$$

which follows from (33).

Starting from

$$
J_{0}(g)=A g^{-1 / 4}
$$

we get

$$
J_{2}^{*}(g)=J_{0}(g) \exp \left(\frac{B}{A} g^{-1 / 2} \exp \left(\frac{C}{B} g^{-1 / 2}\right)\right) .
$$

At the next step, according to (29), we have

$$
F_{3}^{*}(g, s, \tau)=\left[\left(J_{2}^{*}(g)\right)^{-\nu}+\gamma g^{s \nu}\right]^{-1 / \nu}
$$

with

$$
\nu=\frac{6}{1-4 s}, \quad \gamma=-\frac{\nu D \tau}{A^{1+\nu}} .
$$

In the weak-coupling limit, Eq. (37) yields

$$
J_{2}^{*}(g) \simeq A g^{-1 / 4} \quad(g \rightarrow 0)
$$

and Eq. (38) gives

$$
F_{3}^{*}(g, s, \tau)=\left(A^{-\nu} g^{\nu / 4}+\gamma g^{s \nu}\right)^{-1 / \nu} .
$$

The latter expression satisfies the limiting condition

$$
\lim _{g \rightarrow 0} F_{3}^{*}(g, s, \tau)=a,
$$


corresponding to (35), if and only if $s=0$ and $\gamma=a$ or, respectively,

$$
\nu=6, \quad \tau=-\frac{A^{7}}{6 a^{6} D} .
$$

Substituting the found s and $\tau$ into (38), we get

$$
J_{3}^{*}(g)=\left[\left(J_{2}^{*}(g)\right)^{-6}+a^{-6}\right]^{-1 / 6}
$$

Using here form (37) and remembering that $a=1$, we finally obtain the self-similar approximation

$$
J_{3}^{*}(g)=\left[1+\frac{g^{3 / 2}}{A^{6}} \exp \left(\frac{-6 B}{A \sqrt{g}} \exp \left(\frac{C}{B \sqrt{g}}\right)\right)\right]^{-1 / 6}
$$

corresponding to $(31)$.

The exponential approximant (40) represents integral (32) with a very good accuracy. The maximal percentage error is $2.7 \%$ occurring at $g=0.1$. And the error for the physically most interesting region of intermediate $g \approx 1$ is only $0.7 \%$.

Consider another simple example evidently illustrating the generality of our method that works when other methods do not. Assume that we need to find an approximation for a bounded function $f(x)$, with $-\infty<x<\infty$, having the following asymptotic properties:

$$
\begin{gathered}
f(x) \simeq 1+x+O\left(x^{2}\right) \quad(x \rightarrow 0), \\
f(x) \simeq e^{x} \quad(x \rightarrow-\infty), \\
f(x) \simeq \frac{1}{x^{\alpha}}, \quad \alpha>0 \quad(x \rightarrow+\infty),
\end{gathered}
$$

where $\alpha$ is an irrational power. To our understanding, Padé technique is principally inappropriate in this case. While in our method, accomplishing the same procedure as is explained above, we easily obtain

$$
f^{*}(x)=\left[\exp \left(-\frac{2}{\alpha} x\right)+x^{2}\right]^{-\alpha / 2} .
$$

\section{STABILITY CONDITIONS}

In order to check the stability of calculational procedure, one has to analyze mapping multipliers [9-11]. Several kinds of multipliers occur in the process of construction of self-similar approximations, each kind being related to the corresponding approximation cascade.

The first approximation cascade, appearing in our investigation is the cascade $\left\{y_{k}\right\}$ composed of transforms (5). The local multipliers for this cascade are defined as

$$
\mu_{k}(\varphi, s) \equiv \frac{\partial}{\partial \varphi} y_{k}(\varphi, s)=\sum_{n=0}^{k} \frac{\left(s+\alpha_{n}\right) a_{n}}{\left(s+\alpha_{0}\right) a_{0}}\left(\frac{\varphi}{a_{0}}\right)^{\left(\alpha_{n}-\alpha_{0}\right) /\left(s+\alpha_{0}\right)} .
$$

The image of multiplier (41) in the $x$-space can be obtained with the use of relation (3), which yields

$$
m_{k}(x, s) \equiv \mu_{k}\left(P_{0}(x, s), s\right)=\frac{\partial P_{k}(x, s) / \partial x}{\partial P_{0}(x, s) / \partial x}=\sum_{n=0}^{k} \frac{\left(s+\alpha_{n}\right) a_{n}}{\left(s+\alpha_{0}\right) a_{0}} x^{\left(\alpha_{n}-\alpha_{0}\right)} .
$$

The trajectory $\left\{y_{k}(\varphi, s)\right\}$ of an approximation cascade $\left\{y_{k}\right\}$ is locally stable [11] at the $k$-step if $\left|\mu_{k}(\varphi, s)\right|<1$. Note that the case of $\left|\mu_{k}\right|=1$ is called neutrally stable and that of $\left|\mu_{k}\right|=0$ can be termed superstable. If $\left|\mu_{k}\right|<1$ for some $s$ and all $\varphi$ from a given domain, then $\left|m_{k}(x, s)\right|<1$ for the same $s$ and all $x$ from a domain defined by relation (4). When the trajectory of a cascade $\left\{y_{k}\right\}$ is locally stable for all $k=0,1,2, \ldots$, that is, when $\left|m_{k}(x, s)\right|<1$ for all 
$k$, then the sequence $\left\{P_{k}(x, s)\right\}$ converges uniformly with respect to $x$. The local stability for all $k=0,1,2, \ldots$ can be called the global stability. The global stability of a trajectory is a sufficient condition for the convergence of the corresponding sequence [11]. But it is not a necessary condition. Thus, the sequence $\left\{P_{k}(x, s)\right\}$ may be convergent, but the cascade trajectory not everywhere locally stable, so that the stability condition $\left|m_{k}(x, s)\right|<1$ becomes valid for all $k$ starting from some $k_{0}$, but for $k<k_{0}$ this condition may be broken for some $k$.

The concept of stability suggests a recipe of defining the control function $s=s_{k}(x)$. The latter can be defined so that to minimize the absolute value of multiplier (42), which can be named the principle of maximal stability [12-14]. Substituting the found $s_{k}(x)$ into $P_{k}(x, s)$, we obtain $P_{k}\left(x, s_{k}(x)\right)$. The renormalized sequence $\left\{P_{k}\left(x, s_{k}(x)\right)\right\}$ can become convergent even if the initial sequence $\left\{P_{k}(x, s)\right\}$ was not. Defining local multipliers for the new sequence, it is necessary to take into account that $P_{k}\left(x, s_{k}(x)\right)$ depends on $x$ explicitly as well as through $s_{k}(x)$. The corresponding multipliers are

$$
m_{k}(x)=\frac{\partial P_{k} / \partial x+\left(\partial P_{k} / \partial s_{k}\right)\left(d s_{k} / d x\right)}{\partial P_{0} / \partial x+\left(\partial P_{0} / \partial s_{k}\right)\left(d s_{k} / d x\right)},
$$

where $P_{k}=P_{k}\left(x, s_{k}\right), s_{k}=s_{k}(x)$, and the partial derivatives mean that another variable is kept fixed. In general, $m_{k}(x)$ differs from (42). But if $s_{k}(x)$ is a slowly varying function of $x$, such that the derivative $d s_{k} / d x$ in $m_{k}(x)$ can be neglected, then $m_{k}(x)$ approximately coincides with $m_{k}\left(x, s_{k}(x)\right)$. The stability condition $\left|m_{k}(x)\right|<1$ for all $k=0,1,2, \ldots$ implies the convergence of the sequence $\left\{P_{k}\left(x, s_{k}(x)\right)\right\}$. Let us stress again that stability is a sufficient condition for convergence, but not necessary. The sequence $\left\{P_{k}\left(x, s_{k}(x)\right)\right\}$ may converge even if the stability condition is not valid for a finite value of $k$. Moreover, the convergence of the sequence $\left\{P_{k}\left(x, s_{k}(x)\right)\right\}$ is not compulsory for us. This sequence is not yet the final product of the procedure but is to undergone the dynamical renormalization involving the evolution integral (7).

After the multiple dynamical renormalization, in the exponential variant we consider here, we come to the sequence $\left\{F_{k}(x, \tau)\right\}$ of terms given by expression (18), with the shorthand notation $\tau \equiv\left\{\tau_{1}, \tau_{2}, \ldots, \tau_{k}\right\}$. The stability of the corresponding trajectory is characterized by the multipliers

$$
M_{k}(x, \tau) \equiv \frac{\partial F_{k}(x, \tau) / \partial x}{\partial F_{1}(x, \tau) / \partial x} .
$$

The stability condition $\left|M_{k}(x, \tau)\right|<1$ for all $k=1,2, \ldots$ guarantees the convergence of the sequence $\left\{F_{k}(x, \tau)\right\}$. This tells us that the time-control functions $\tau_{k}(x)$ could be chosen so that to minimize $\left|M_{k}(x, \tau)\right|$. In particular, the minimal value of (44) can be zero. Then the equality $M_{k}(x, \tau)=0$, under the assumption that $\partial F_{1}(x, \tau) / \partial x \neq 0$, yields the equation

$$
\frac{\partial}{\partial x} F_{k}(x, \tau)=0
$$

restricting the choice of time-control functions.

Defining all time-control functions by one of the ways discussed above, we obtain, as a final result, the exponential approximant (20). The sequence $\left\{f_{k}^{*}(x)\right\}$, with $k=1,2, \ldots$, of these exponential approximants is what we ultimately need to analyze with respect to its convergence. For that purpose we can formulate sufficient conditions for convergence studying the stability of the corresponding cascade. To this end, following the standard procedure [7-11], we define a function $x(\varphi)$ by the equation

$$
f_{1}^{*}(x)=\varphi, \quad x=x(\varphi) .
$$

Then we introduce the transformation

$$
y_{k}^{*}(\varphi)=f_{k}^{*}(x(\varphi)) .
$$

The family $\left\{y_{k}^{*}\right\}$ of the transformations introduced in (47) forms a cascade. The local multipliers are given by

$$
\mu_{k}^{*}(\varphi) \equiv \frac{\partial}{\partial \varphi} y_{k}^{*}(\varphi)
$$

The image of (48) in the $x$-representation is

$$
m_{k}^{*}(x) \equiv \mu_{k}^{*}\left(f_{1}^{*}(x)\right)=\frac{\partial f_{k}^{*}(x) / \partial x}{\partial f_{1}^{*}(x) / \partial x} .
$$


The cascade trajectory is locally stable at a $k$-step when

$$
\left|m_{k}^{*}(x)\right|<1 .
$$

If this condition is valid for all $k$, then the sequence $\left\{f_{k}^{*}(x)\right\}$ converges.

The validity of the stability condition (50) is what finally justifies the whole renormalization procedure guaranteeing the convergence of the renormalized sequence $\left\{f_{k}^{*}(x)\right\}$. This holds true irrespectively of whether other multipliers, as (42), (43), or (44), satisfy the same condition (50) as the multiplier (49). The auxiliary multipliers (42)-(44) have appeared at intermediate stages of our multistep renormalization procedure. The minimization of these multipliers provides a recipe for defining control functions. This minimization is equivalent to the stabilization of local parts of a cascade trajectory. Remind that we start with series (1) which, in general, is strongly divergent. Accomplishing several stages of renormalization we step by step improve convergence properties, by making the related trajectory more and more stabilized. However, it is not necessary to demand that the trajectory would become completely stable at some intermediate step of the multistep procedure. The main is that the finally resulting sequence $\left\{f_{k}^{*}(x)\right\}$ be convergent, for which it is sufficient to require the validity of condition (50).

\section{GROUND STATE PROPERTIES}

Now we pass to the consideration of physical examples illustrating explicitly how the method works.

\section{A. One-Dimensional Bose System}

The ground-state energy of the one-dimensional Bose system with the $\delta$-functional repulsive interaction potential is known in a numerical form from the Lieb-Liniger exact solution [42]. We derive below a compact analytical expression for the ground-state energy $e(g)$ as a function of the interaction strength $g$, valid for arbitrary $g$. In the weak-coupling and strong-coupling limits the following expansions are known (see e.g. Ref.[7] and references therein) :

$$
\begin{aligned}
& e(g) \simeq a g+b g^{3 / 2}+c g^{2}+d g^{5 / 2}+\ldots, \quad(g \rightarrow 0), \\
& a=1, \quad b=-\frac{4}{3 \pi}, \quad c=0.0654, \quad d=-0.0018,
\end{aligned}
$$

while in the strong-coupling limit an exact result is available [43]:

$$
e(g) \simeq A=\frac{\pi^{2}}{3}, \quad(g \rightarrow \infty) .
$$

The expression satisfying both known limits, can be derived similarly to the example studied in Section III, except that in this case we start from the weak-coupling limit. The resulting approximant is

$$
e_{4}^{*}(g)=a g\left[\left(\exp \left(\frac{b}{a} g^{1 / 2} \exp \left(\frac{c}{b} g^{1 / 2}\right)\right)\right)^{-3 / 2}+\left(\frac{A}{a}\right)^{-3 / 2} g^{3 / 2}\right]^{-2 / 3} .
$$

This expression works reasonably well in the region of $g \in[0,10]$, where the exact numerical solution of the Bethe ansatz equations is available. The maximal error here is about $5 \%$.

\section{B. Asymmetric Anderson Hamiltonian}

The single-orbital Anderson Hamiltonian describes the system consisting of localized $d$-electrons interacting with conducting $s$-electrons via a quantum-mechanical exchange mechanism, whose strength is measured by $V$, which is the transfer integral between the $s$ - and $d$-states. The energy $U$ describing the Coulomb interactions between two $d$-electrons is another relevant physical parameter. The localized $d$-electron number $n_{d}$ is expressed through the self-energy $\Sigma$ of $d$-electrons at the Fermi level: 


$$
n_{d}=\frac{1}{2}-\frac{1}{\pi} \tanh ^{-1}\left(\frac{\Sigma}{\Delta}\right), \quad \Delta=\pi \rho V^{2},
$$

where $\rho$ stands for the density of states at the Fermi level for conducting electrons. In the case when $d$-level is fixed to the Fermi-level, the following expansion for $\Sigma$, in powers of the small parameter $u=U / \pi \Delta,|u| \ll 1$, was obtained in Ref. [44]:

$$
\begin{gathered}
\frac{\Sigma}{\Delta} \simeq \frac{\pi}{2} u\left(1+a_{1} u+a_{2} u^{2}+a_{3} u^{3}+a_{4} u^{4}+a_{5} u^{5}\right) \quad(u \rightarrow 0), \\
a_{1}=-1, \quad a_{2}=0.5326, \quad a_{3}=0.6269, \quad a_{4}=-1.8071, \quad a_{5}=1.027 .
\end{gathered}
$$

A direct application of expansion (55), gives physically meaningful results in the region of $-0.5<u<0.5$, as is shown in Fig.2 of Ref.[44]. However, the exactly known limits

$$
n_{d} \rightarrow 0 \quad(u \rightarrow \infty) ; \quad n_{d} \rightarrow 1 \quad(u \rightarrow-\infty)
$$

are violated when expansion (55) is used for the calculation of the impurity level occupancy (54). To our knowledge, the Padé approximants were not applied for resumming expansion (55), and it is not clear whether they can be applied at large values of the parameter $u$ (see $[2,45]$ ). We use below the technique of exponential approximants and observe that they spontaneously recover the known limits. The following sequence of self-similar renormalized expressions can be written:

$$
\begin{gathered}
\left(\frac{\Sigma}{\Delta}\right)_{1}^{*}=\frac{\pi}{2} u\left(\exp \left(a_{1} u\right)\right), \\
\left(\frac{\Sigma}{\Delta}\right)_{2}^{*}=\frac{\pi}{2} u\left[\exp \left(a_{1} u \exp \left(\frac{a_{2}}{a_{1}} u\right)\right)\right], \\
\left(\frac{\Sigma}{\Delta}\right)_{3}^{*}=\frac{\pi}{2} u\left[\exp \left(a_{1} u \exp \left(\frac{a_{2}}{a_{1}} u \exp \left(\frac{a_{3}}{a_{2}} u\right)\right)\right)\right], \\
\left(\frac{\Sigma}{\Delta}\right)_{4}^{*}=\frac{\pi}{2} u\left[\exp \left(a_{1} u \exp \left(\frac{a_{2}}{a_{1}} u \exp \left(\frac{a_{3}}{a_{2}} u \exp \left(\frac{a_{4}}{a_{3}} u\right)\right)\right)\right)\right] \\
\left(\frac{\Sigma}{\Delta}\right)_{5}^{*}=\frac{\pi}{2} u\left[\exp \left(a_{1} u \exp \left(\frac{a_{2}}{a_{1}} u \exp \left(\frac{a_{3}}{a_{2}} u \exp \left(\frac{a_{4}}{a_{3}} u \exp \left(\frac{a_{5}}{a_{4}} u\right)\right)\right)\right)\right)\right] .
\end{gathered}
$$

We observe, that already the third-order approximant $\left(\frac{\Sigma}{\Delta}\right)_{3}^{*}$ leads to the result

$$
\left(n_{d}\right)_{3}^{*}=\frac{1}{2}-\frac{1}{\pi} \tanh ^{-1}\left[\left(\frac{\Sigma}{\Delta}\right)_{3}^{*}\right]
$$

which possesses the correct limits. The same is true when the higher order approximants

$$
\left(n_{d}\right)_{4}^{*}=\frac{1}{2}-\frac{1}{\pi} \tanh ^{-1}\left[\left(\frac{\Sigma}{\Delta}\right)_{4}^{*}\right], \quad\left(n_{d}\right)_{5}^{*}=\frac{1}{2}-\frac{1}{\pi} \tanh ^{-1}\left[\left(\frac{\Sigma}{\Delta}\right)_{5}^{*}\right],
$$

are considered. An aposteriori analysis of multipliers suggests that the third-order approximant corresponds to the most stable trajectory. This could be expected beforehand, since the coefficients $a_{4}$ and $a_{5}$ are larger than one.

The density of states of $d$-electrons as well as the resistivity $R$ at zero temperature, could be also reconstructed for arbitrary $u$, e.g.

$$
\left(\frac{R(u)}{R(0)}\right)^{*}=\left[1+\left(\left(\frac{\Sigma}{\Delta}\right)_{3}^{*}\right)^{2}\right]^{-1}
$$

The shape of the curve $\left(n_{d}(u)\right)_{3}^{*}$ is very much like the smeared Fermi-distribution, while $\left(\frac{R(u)}{R(0)}\right)^{*}$ has an asymmetric bell-shape. All artifacts, which appear at the curves, when the perturbative expansions are naively extended beyond the region of small $u$, are smeared out, and the renormalized curves appear to be rather smooth. 


\section{C. t-expansion}

The so-called $t$-expansion is a tool for a systematic improvement of variational calculations for Hamiltonian systems [46]. Using a $t$-dependent variational wave function and, after performing calculations, finally, taking the $t \rightarrow \infty$ limit, one can hope to increase the quality of the variational estimate for the ground-state energy $E$. This idea is most frequently used in conjunction with heavy numerical calculations and Padé-approximants technique. In the case of the $1 d$ Heisenberg antiferromagnet, the expansion in powers of the parameter $t$ was obtained explicitly [46],

$$
E \simeq-\frac{1}{4}-t+2 t^{2}+\frac{4}{3} t^{3}-16 t^{4} \quad(t \rightarrow 0),
$$

and the task of getting an estimate for the ground state energy from the asymptotic expression valid at $t \rightarrow 0$, can be approached by the methods of Sections II-IV. Since the number of terms available is finite, it is not necessary to take the limit $t \rightarrow \infty$ explicitly, but instead we can try to minimize the error caused by this inevitable truncation by demanding the minimal sensitivity of the renormalized expression $E^{*}$ with respect to the "time" $t$ determining the "duration" of motion to the ground state energy,

$$
\frac{\partial E^{*}}{\partial t}=0
$$

Applying the self-similar bootstrap in its super-exponential form, one can see that the solution to this equation does exist for any number of terms from the initial expansion (62). From the aposteriori analysis of the multipliers, we conclude that the most reliable value, corresponding to that obtained along the most stable trajectory, is

$$
E^{*}=-\frac{1}{4} \exp \left(4 t \exp \left(-2 t \exp \left(\frac{2}{3} t\right)\right)\right)
$$

with $t=0.33$ and $E^{*}=-0.446$. From the viewpoint of an apriori analysis, it is admissible also to construct another sequence of exponentials, not including the constant, mean-field result. In this case, the answer for the ground state energy is -0.434 . We conclude that moving along the two different, but stable, trajectories, we can determine $E$ reliably, as $E=-0.44 \pm 0.006$. The known Hulthen [47] exact result -0.4431 is located within these boundaries.

\section{EFFECTIVE COUPLING}

\section{A. Beta-Function of SU(2) Lattice Gauge Model}

The Callan-Symanzik $\beta$-function of the (3+1)-dimensional SU(2) lattice gauge model in its weak-coupling asymptotically free regime may be presented in the form of an expansion in powers of the parameter $g$, where $g$ stands for the coupling [48]:

$$
\begin{gathered}
-\frac{\beta(g)}{g} \simeq a g^{2}+b g^{4}+\ldots, \quad(g \rightarrow 0), \\
a=\frac{11}{24 \pi^{2}}, \quad b=\frac{17}{64 \pi^{4}} .
\end{gathered}
$$

In its strong-coupling limit, $\beta$ can be presented as follows [48]:

$$
\begin{gathered}
-\frac{\beta(g)}{g} \simeq A+B x^{2}+C x^{4}+D x^{6}+F x^{8}, \quad x=\frac{4}{g^{4}} \quad(g \rightarrow \infty), \\
A=1, \quad B=-\frac{76}{75}, \quad C=\frac{88}{625}, \quad D=-\frac{131203}{140625}, \quad F=\frac{551378}{390625} .
\end{gathered}
$$

We will add to the expansion (66) one more trial term $\simeq G x^{10}$, and determine $G$ from the boundary condition following from the weak-coupling limit (65). Two starting terms from (66) will be renormalized to the form of an exponential approximant, mimicking an instanton contribution bridging these two limits [49], that is, a nonperturbative physical 
mechanism coming into play in the crossover region, from strong-to-weak-coupling limit [50]. This term should disappear completely in the weak-coupling limit, guarantying rather sharp crossover. The last four terms will be renormalized to the form satisfying the boundary condition (65), in a way leading to a smooth matching of two limiting kinds of behavior. We obtain the following analytical form for the renormalized $\beta$-function:

$$
\begin{gathered}
-\frac{\beta^{*}(g)}{g}=A \exp \left(\frac{B}{A} x^{2}\right)+C x^{4}\left(1-\frac{\tau_{1}}{s_{1}} \frac{D}{C} x^{2}\right)^{-s_{1}}+F x^{8}\left(1-\frac{G}{s_{2} F} x^{2}\right)^{-s_{2}}, \\
s_{1}=\frac{9}{4}, \quad \tau_{1}=-\frac{s_{1} C}{D}\left(\frac{2 a}{C}\right)^{-1 / s_{1}}=0.3 ; \quad s_{2}=2 s_{1}, \quad G=-s_{2} F\left(\frac{4 b}{F}\right)^{-1 / s_{2}}=-15.051 .
\end{gathered}
$$

The shape of the resulting function is similar to those obtained in Refs.[48-50]. Crossover occurs at $g \sim 1.2$. Let us remind that the quality of the strong-coupling expansions is jeopardized by the interfering roughening transition [48]. We believe that the method discussed above, allows us to bypass this difficulty in a natural way.

\section{B. Kondo Effect}

We consider below an application of the exponential approximants to such an interesting problem as the Kondo effect [51], comparing our results with those of the field-theoretical renormalization group [52-54].

The behavior of the system, consisting of a local-impurity spin and conduction electrons, interacting by means of an antiferromagnetic exchange of strength $J$, changes from asymptotically free at high temperatures to that when the impurity is screened by electronic lump at low temperatures, via the crossover region whose onset is characterized by the Kondo temperature estimated as

$$
T_{k}=D \exp \left(-\frac{1}{2 J}\right)
$$

where $D$ stands for the Fermi-energy of electrons. We consider below only the case of a single-channel Kondo model. Most of our knowledge about the problem came from the exact Bethe ansatz solution [55,56], from the field-theoretical renormalization group [52-54], and from the Wilson numerical renormalization group [57].

Within the framework of the field-theoretical RG in its application to the Kondo crossover, the central role is played by the so-called invariant charge or effective electron-electron coupling $J_{i n v}$ [52-54], measuring the intensity of electron-electron interactions via the impurity spin:

$$
J_{i n v}=J\left[1+2 J \ln \left(\frac{D}{|\omega|}\right)-2 J^{2} \ln \left(\frac{D}{|\omega|}\right)+\ldots\right],
$$

here $\omega$ stands for the typical external parameter of the problem, such as temperature, or magnetic field. If only the starting two terms from (69) are taken into account, the field-theoretical approach, through calculations based on the Gell-Mann-Low $\beta$-function,

$$
\beta \simeq-2 J^{2}, \quad(J \ll 1),
$$

leads to the formally divergent, at $T=T_{k}$, expression for the invariant charge [52-54],

$$
J_{i n v}=\frac{J}{1-2 J \ln \left(\frac{D}{|\omega|}\right)} .
$$

We apply below the technique of algebraic self-similar renormalization directly to the series (69) for $J_{i n v}$, continuing them from the region of $J \ll 1$ to the region of $J \sim 1$. When only two starting terms from (69) are taken into account, the optimal, from the viewpoint of stability conditions, solution is the following exponential approximant,

$$
J_{i n v}^{*}=J \exp \left[2 J \ln \left(\frac{D}{|\omega|}\right)\right]=J\left(\frac{D}{|\omega|}\right)^{2 J} .
$$


If we perform the self-similar renormalization with the control function $s=0$, along the non-optimal trajectory, we will recover the expression (71). So, the fictitious pole is absent in our solution to the problem, although the typical energy scale determined from the condition

$$
2 J \ln \left(\frac{D}{|\omega|}\right) \sim 1,
$$

coincides with the Kondo temperature (68). Expression (72) is formally divergent as $\omega \rightarrow 0$, in agreement with the conclusion of the numerical renormalization group [57,58].

More complicated situation arises in (quasi)-two-dimensional metal, when the Van Hove logarithmic singularity in the electron density of states can influence the Kondo effect. In this case, as was shown in Ref.[59], the full electron-impurity scattering amplitude $\Gamma$ can be estimated as follows:

$$
\Gamma=J\left[1+c_{0} J\left(\ln \left(\frac{D}{|\omega|}\right)\right)^{2}+\ldots\right], \quad c_{0}>0, \quad(J \ll 1),
$$

i.e. the usual Kondo logarithm should be replaced by the squared logarithm, originating from the Van Hove singularity. The self-similarly renormalized expression in this case again corresponds to the exponential approximant:

$$
\Gamma=J \exp \left[c_{0} J\left(\ln \left(\frac{D}{|\omega|}\right)\right)^{2}\right],
$$

and the characteristic energy scale, Kondo temperature, can be found from the condition

$$
c_{0} J\left(\ln \left(\frac{D}{|\omega|}\right)\right)^{2} \sim 1,
$$

leading to the estimate

$$
T_{k}=D \exp \left(-\frac{\text { const }}{\sqrt{J}}\right), \quad \text { const } \sim \sqrt{\frac{1}{c_{0}}} .
$$

Such a dependence of $T_{k}$ on $J$ was the main result of Ref.[59], obtained as an outcome of a cumbersome first order parquet summation. To our knowledge, the field-theoretical RG approach was not applied to the Kondo effect with the Van Hove singularity. On the other hand, the Bethe ansatz fails for this problem [59].

The higher-order corrections were not considered in Ref.[59], because of the technical difficulties arising in more sophisticated parquet approximations. Our approach may be of interest in this context, allowing to find the corrections to Kondo temperature due to higher-order terms. Taking into account the higher-order perturbative terms, one has

$$
\Gamma=J\left[1+c_{0} J\left(\ln \left(\frac{D}{|\omega|}\right)\right)^{2}+c_{1} J^{2}\left(\ln \left(\frac{D}{|\omega|}\right)\right)^{2}+\ldots\right], \quad c_{0}>0, \quad(J \ll 1),
$$

with $c_{1}<0$. The following exponential approximant is optimal from the viewpoint of stability:

$$
\Gamma=J \exp \left[c_{0} J\left(\ln \left(\frac{D}{|\omega|}\right)\right)^{2} \exp \left(-\frac{\left|c_{1}\right|}{c_{0}} J\right)\right] .
$$

From the condition

$$
c_{0} J\left(\ln \left(\frac{D}{|\omega|}\right)\right)^{2} \exp \left(-\frac{\left|c_{1}\right|}{c_{0}} J\right) \sim 1,
$$

we obtain the Kondo temperature:

$$
T_{k}=D \exp \left[-\frac{1}{c_{0}^{1 / 2} J^{1 / 2}} \exp \left(\frac{1}{2} \frac{\left|c_{1}\right|}{c_{0}} J\right)\right] .
$$

This estimate suggests a decrease of $T_{k}$ due to higher-order corrections. 


\section{EQUATION OF STATE}

\section{A. Classical Hard Spheres.}

The exponential approximants can be used for constructing equations of state for simple liquids. For the model system of hard spheres with the diameter $d$, widely used as a reference system, an empirical equation of state, suggested by Carnagan and Starling (see [60]), is

$$
\frac{p}{n k T}=\frac{1+\rho+\rho^{2}-\rho^{3}}{(1-\rho)^{3}}
$$

connecting pressure $p$, temperature $T$, the number density $n$, and the reduced density $\rho=\pi n d^{2} / 6$. It agrees very well with the molecular dynamics and virial expansion [60,61]. The theoretical virial formula, according to Percus-Yevick $[60,61]$, is given as follows:

$$
\frac{p}{n k T}=\frac{1+\rho+\rho^{2}-3 \rho^{3}}{(1-\rho)^{3}} .
$$

These two expressions almost coincide at low densities, e.g. at $\rho=0.1$ the percentage error of Eq.(80), as compared to (79), equals $-0.18 \%$; while for the intermediate and high densities the agreement becomes very poor, e.g. at $\rho=0.5$ the percentage error is $-15.385 \%$, and at $\rho=0.7$ it equals $-37.141 \%$.

Consider the regular part of (80), defined as $r$ :

$$
r \simeq 1+\rho+\rho^{2}-3 \rho^{3} \quad(\rho \rightarrow 0)
$$

as an asymptotic, low-density expansion for the regular part $r(\rho)$, and try to continue the expression (81) from the region of $\rho \ll 1$, to the region of $\rho \leq 1$. In order to extend the validity of $(81)$, let us add to it one more trial term $\sim \rho^{4}$. It is reasonable to use for renormalization the last four terms from thus extended expansion for $r$, since the constant term describes the ideal gas behavior and we are interested in the region of high densities. Following the standard prescriptions of Sec.II-III, we write down the two exponential approximants, justified from the viewpoint of stability for the sequence of an aposteriori multipliers:

$$
\begin{gathered}
r_{3}^{*}(\rho, \tau)=1+\rho \exp [\rho \exp (-3 \rho \tau)] \\
r_{4}^{*}(\rho, \tau)=1+\rho \exp \left[\rho \exp \left(-3 \rho \exp \left(-\frac{\tau}{3} \rho\right)\right)\right] .
\end{gathered}
$$

We retained in expressions (82) and (83) the effective time $\tau$, introduced at the last step of the bootstrap procedure. It will work now as a control function $\tau=\tau(\rho)$ determined from the minimal-velocity condition $\min _{\tau}\left|r_{4}^{*}(\rho, \tau)-r_{3}^{*}(\rho, \tau)\right|$. For the sake of simplicity we choose a single control parameter $\tau$, instead of the control function, from the minimal difference condition imposed at a single point, chosen from the region of intermediate densities, say for $\rho=0.6$. Then, $\tau=0.845$. Recalculating

$$
\frac{p^{*}}{n k T}=\frac{r_{4}^{*}(\rho, \tau)}{(1-\rho)^{3}},
$$

and comparing it with empirical formula (79), we obtain that at $\rho=0.1$ the percentage error equals $-0.112 \%$; at $\rho=0.5$ the percentage error is $-3.215 \%$, and at $\rho=0.7$ it equals $-2.92 \%$. Formula (84) is more accurate at high densities than our previous result [14], corresponding to

$$
\frac{p^{*}}{n k T}=\frac{r_{3}^{*}(\rho, 1)}{(1-\rho)^{3}},
$$

which works with the percentage error of $-4.567 \%$ at $\rho=0.7$. What is even more important here, is the possibility of a self-consistent improvement of the quality of the equation of state, based on stability conditions. We should recall here, that phenomenological exponential-type expressions are well known in the theory of equations of state, beginning, probably, from the Hudleston equation (see [61] and references therein) and ending with its modern modifications [62-64]. For example, the Shinomoto's equation for the system of hard spheres reads: 


$$
\frac{p}{n k T}=\exp \left[4 \rho\left(1+\frac{1}{2} \rho\right)\right]
$$

and gives at $\rho=0.1$ the percentage error $0.046 \%$; at $\rho=0.5$ the error is $-6.289 \%$, and at $\rho=0.7$ it becomes $-35.948 \%$.

A single-exponential approximation can also be obtained from Eq.(81) by our method:

$$
\frac{p^{*}}{n k T}=\frac{\rho \exp \left(\rho-3 \rho^{2}\right)+1}{(1-\rho)^{3}}
$$

giving at $\rho=0.1$ the error $-0.158 \%$, at $\rho=0.5$ the error is $-14.498 \%$, and at $\rho=0.7$ it becomes $-28.31 \%$.

We conclude that the multi-exponential formula (84) agrees well with the empirical formula (79), being superior to all other formulae in the region of high densities.

\section{B. Quantum Hard Spheres}

At low density $\rho$, the energy $E$ for a boson system of $N$-hard spheres with the diameter $c$ and mass $m$ is known $[65]$ :

$$
\frac{E}{N} \simeq \frac{2 \pi}{m} \rho c\left(1+C_{1}\left(\rho c^{3}\right)^{1 / 2}+\ldots\right), \quad C_{1}=\frac{128}{15 \sqrt{\pi}} \quad(\rho \rightarrow 0)
$$

And as $\rho \rightarrow \rho_{0}$, where $\rho_{0}=\sqrt{2} / c^{3}$ is the maximal density for a system of hard spheres, the following expression is available [65]:

$$
\frac{E}{N} \simeq A \frac{1}{2 m}\left(\rho^{-1 / 3}-\rho_{0}^{-1 / 3}\right)^{-2}, \quad A=\frac{\pi^{2}}{2^{1 / 3}} \quad\left(\rho \rightarrow \rho_{0}\right),
$$

corresponding to a second-order pole in the ground-state energy. Let us, in analogy with the previous example, extract the singularity, as $\rho \rightarrow \rho_{0}$, rewriting (88) as follows:

$$
\frac{E}{N} \simeq \frac{2 \pi c}{m} \frac{\rho^{1 / 3}\left[1+C_{1}\left(\rho c^{3}\right)^{1 / 2}+\ldots\right]}{\left(\rho^{-1 / 3}-\rho_{0}^{-1 / 3}\right)^{2}}\left[1-\left(\frac{\rho}{\rho_{0}}\right)^{1 / 3}\right]^{2}, \quad(\rho \rightarrow 0),
$$

and, keeping only a few starting terms

$$
\frac{E}{N} \simeq \frac{2 \pi c}{m} \frac{\rho^{1 / 3}}{\left(\rho^{-1 / 3}-\rho_{0}^{-1 / 3}\right)^{2}}\left[1-2\left(\frac{\rho}{\rho_{0}}\right)^{1 / 3}+C_{1}\left(\rho c^{3}\right)^{1 / 2}+\ldots\right] .
$$

After the standard self-similar renormalization, involving two terms from (91), we obtain

$$
\frac{E^{*}}{N}=\frac{2 \pi c}{m} \frac{\rho^{1 / 3}}{\left(\rho^{-1 / 3}-\rho_{0}^{-1 / 3}\right)^{2}} \exp \left[-2 \tau\left(\frac{\rho}{\rho_{0}}\right)^{1 / 3}\right]
$$

where the control parameter $\tau$ should be determined from the known asymptotic formula (89), as $\rho \rightarrow \rho_{0}$. Finally,

$$
\frac{E^{*}}{N}=\frac{2 \pi c}{m} \frac{\rho^{1 / 3}}{\left(\rho^{-1 / 3}-\rho_{0}^{-1 / 3}\right)^{2}} \exp \left[\ln \left(\frac{A}{4 \pi c} \rho_{0}^{-1 / 3}\right)\left(\frac{\rho}{\rho_{0}}\right)^{1 / 3}\right], \quad \tau=-\frac{1}{2} \ln \left(\frac{A}{4 \pi c} \rho_{0}^{-1 / 3}\right),
$$

or, equivalently,

$$
\frac{E^{*}}{N}=\frac{2 \pi c}{m} \frac{\rho^{1 / 3}}{\left(\rho^{-1 / 3}-\rho_{0}^{-1 / 3}\right)^{2}}\left(\frac{A}{4 \pi c} \rho_{0}^{-1 / 3}\right)^{\left(\rho / \rho_{0}\right)^{1 / 3}}
$$

Equations (93) and (94) should be compared with the empirical London equation of state [66]. Both equations give the results very close to each other. So, our derivation can serve as a justification for empirical formulae used for the system of Bose hard spheres. 


\section{Polymer Coil}

The expansion factor $\alpha$ of a polymer coil is represented as a function $\alpha^{2}=\alpha^{2}(z)$ of the excluded volume variable $z=B \sqrt{N}\left(3 / 2 \pi l^{2}\right)^{3 / 2}$, where $N$ is the number of bonds of the length $l$ each and $B$ is an effective binary cluster integral [67-70]. The case of a polymer coil corresponds to $z>0$. In the region of $z \ll 1$, the perturbation theory in powers of $z$ can be developed, giving the expansion [68]

$$
\begin{gathered}
\alpha^{2}=\alpha^{2}(z) \simeq 1+k_{1} z+k_{2} z^{2}+k_{3} z^{3}+k_{4} z^{4}+k_{5} z^{5}+k_{6} z^{6} \quad(z \rightarrow 0), \\
k_{1}=\frac{4}{3}, \quad k_{2}=-2.075385396, \quad k_{3}=6.296879676 \\
k_{4}=-25.05725072, \quad k_{5}=116.134785, \quad k_{6}=-594.71663
\end{gathered}
$$

On the other hand, in the limit of $z \gg 1, \alpha$ is related to $z$ by a simple power law

$$
\alpha^{2} \simeq K z^{b}, \quad b=2(2 \nu-1) \quad(z \rightarrow \infty),
$$

where $\nu$ is the critical index, and $K$ stands for the critical amplitude. One of the popular problems in the physics of polymer coils consists in the continuation of the expansion (95) to the region of arbitrary $z$. We derive below, using the self-similar renormalization, a simple equation of state for the polymer coil, valid for arbitrary $z$, and satisfying by design both known limits, (95) and (96). The coefficients in expansion (95), starting from $k_{3}$, grow rapidly, so do the local multipliers. Because of this, we use only three starting terms from (95), stabilizing the renormalized expression by imposing the asymptotic condition (96). Finally, after the standard transformations analogous to those of Section III, we obtain

$$
\left(\alpha^{2}(z)\right)^{*}=\left[\left(\exp \left(k_{1} z \exp \left(\frac{k_{2}}{k_{1}} z\right)\right)\right)^{3 / b}+K^{3 / b} z^{3}\right]^{b / 3}
$$

It is known, from different approaches [12,69,70], that $1 / 2 \leq \nu \leq 0.6$ and $1.53 \leq K \leq 1.75$. We take the values $\nu=0.599$ and $K=1.62$, which we calculated in Ref.[12], and compare the equation of state (97) with the empirical equations of state [70] of Barrett-Domb,

$$
\alpha^{2}=\left(1+\frac{20}{3} z+4 \pi z^{2}\right)^{1 / 5}
$$

and of Yamakawa-Tanaka,

$$
\alpha^{2}=0.572+0.428(1+6.23 z)^{1 / 2}
$$

In the region $0 \leq z \leq 12$, our equation (97) yields a curve lying between those of eqs.(98) and (99).

\section{Ising Model}

The low-temperature expansion for the order parameter (magnetization) $M$ of the three-dimensional Ising model on a f.c.c. lattice reads [71]:

$$
\begin{gathered}
M(T) \simeq 1+b u^{6}+c u^{11}, \quad u=\exp \left(-\frac{4}{T}\right) \quad(T \rightarrow 0), \\
b=-2, \quad c=-24 .
\end{gathered}
$$

Low-temperature expansions are non-universal in the sense that they depend on the type of a lattice, spin etc.... At the critical point $T_{c}$, order parameter demonstrates a universal behavior, independent on the type of a lattice, spin etc...: 


$$
M \sim\left(T_{c}-T\right)^{\beta}, \quad\left(T \rightarrow T_{c}\right)
$$

where $\beta \approx 0.325$ is the critical index [72]. For the f.c.c. lattice, $T_{c} \approx 9.8[72]$. We continue the low-temperature expansion (100) self-similarly to the whole region of $0 \leq T \leq T_{c}$, imposing relation (101) as a boundary condition. Following the standard prescriptions of Section III, we obtain

$$
\begin{gathered}
M^{*}(T)=\left[\left(\exp \left(b u^{6}\right)\right)^{-11 / s}-\frac{11 c}{s} \tau u^{11}\right]^{-s / 11}, \\
s=-11 \beta, \quad \tau=-\frac{\beta}{c u^{11}\left(T_{c}\right)} \exp \left(\frac{b}{\beta} u^{6}\left(T_{c}\right)\right)=0.718 .
\end{gathered}
$$

In the region of intermediate temperatures, Eq. (102) agrees much better with the experimental data for the magnetization of $\mathrm{Fe}$ and $\mathrm{Ni}$ [73] than the Bragg-Williams approximation. In distinction from the Burley extrapolation [74], our formula is simple and has a transparent physical background, taking into account both short-and-middle-range correlations, which contribute through the exponential function at low and intermediate temperatures, as well as long-range correlations dominating at the critical point.

\section{MAGNETIC PROPERTIES}

The ground state properties of the two-dimensional Heisenberg antiferromagnet can be considered by means of an expansion around its Ising limit in powers of the anisotropy parameter $x$, equal to zero for the Ising model and equal to one for the Heisenberg-limit $[75,76]$. Such expansions can be generated separately for the most interesting cases of spin-1/2 and spin-1, allowing thus to treat them independently, not relying on the expansion in inverse powers of spin around a very distant case of classical spins.

The expression for susceptibility $\chi$ of the two-dimensional Heisenberg antiferromagnet was obtained in the following form $[75,76]$ :

$$
\begin{gathered}
\frac{1}{2} \chi \simeq \frac{1}{8}+a_{1} x+a_{2} x^{2}+a_{3} x^{3}+a_{4} x^{4} \quad(x \rightarrow 0), \\
a_{1}=-\frac{1}{6}, \quad a_{2}=0.177083, \quad a_{3}=-0.1898148, \quad a_{4}=0.191761, \quad a_{5}=-0.196579 \\
a_{6}=0.197934, \quad a_{7}=-0.201447 \quad\left(S=\frac{1}{2}\right) ; \\
a_{1}=-0.142857, \quad a_{2}=0.144643, \quad a_{3}=-0.149916, \quad a_{4}=0.150672, \quad a_{5}=-0.153095 \\
a_{6}=0.153437, \quad a_{7}=-0.154932 \quad(S=1) .
\end{gathered}
$$

According to general prescriptions of Section IV, we should, first, analyze the values of local multipliers $m_{k}(s)$, as $s \rightarrow \infty$.

Consider the case of $S=1$. Since the values of the coefficients in the expansion (103) are slowly growing and oscillate with increasing number, the local multipliers will oscillate too. We conclude that in some cases we can continue the trajectory moving along the stable regions, while in other cases we have to move along unstable regions. In this situation we must rely on the aposteriori analysis. In order to choose the starting term in (103), let us compare the values of $m_{k}(s)$, as $s \rightarrow \infty$. Then $m_{1}(\infty)=1+a_{2} / a_{1}=-0.143$ and $m_{2}(\infty)=1+a_{3} / a_{2}=-0.013$. Since the latter number is smaller, we should start the renormalization procedure from the linear term, keeping the constant term untouched. The following sequence of exponential approximants can be readily written down, with the corresponding multipliers shown in brackets, calculated at $\tau=1$ and $x=1$ :

$$
\frac{1}{2} \chi_{2}^{*}(\tau)=\frac{1}{8}+a_{1} x \exp \left(\frac{a_{2}}{a_{1}} x \tau\right)=0.146 \quad\left(m_{1}^{*}=1\right)
$$




$$
\begin{aligned}
& \frac{1}{2} \chi_{3}^{*}(\tau)=\frac{1}{8}+a_{1} x \exp \left[\frac{a_{2}}{a_{1}} x \exp \left(\frac{a_{3}}{a_{2}} x \tau\right)\right]=0.05 \quad\left(m_{2}^{*}=-0.025\right), \\
& \frac{1}{2} \chi_{4}^{*}(\tau)=\frac{1}{8}+a_{1} x \exp \left[\frac{a_{2}}{a_{1}} x \exp \left(\frac{a_{3}}{a_{2}} x \exp \left(\frac{a_{4}}{a_{3}} x \tau\right)\right)\right]=0.107 \quad\left(m_{3}^{*}=0.944\right), \\
& \frac{1}{2} \chi_{5}^{*}(\tau)=\frac{1}{8}+a_{1} x \exp \left[\frac{a_{2}}{a_{1}} x \exp \left(\frac{a_{3}}{a_{2}} x \exp \left(\frac{a_{4}}{a_{3}} x \exp \left(\frac{a_{5}}{a_{4}} x \tau\right)\right)\right)\right]=0.075 \\
& \left(m_{4}^{*}=0.225\right) \\
& \frac{1}{2} \chi_{6}^{*}(\tau)=\frac{1}{8}+a_{1} x \exp \left[\frac{a_{2}}{a_{1}} x \exp \left(\frac{a_{3}}{a_{2}} x \exp \left(\frac{a_{4}}{a_{3}} x \exp \left(\frac{a_{5}}{a_{4}} x \exp \left(\frac{a_{6}}{a_{5}} x \tau\right)\right)\right)\right)\right]=0.094 \\
& \left(m_{5}^{*}=0.754\right) \\
& \frac{1}{2} \chi_{7}^{*}(\tau)=\frac{1}{8}+ \\
& +a_{1} x \exp \left[\frac{a_{2}}{a_{1}} x \exp \left(\frac{a_{3}}{a_{2}} x \exp \left(\frac{a_{4}}{a_{3}} x \exp \left(\frac{a_{5}}{a_{4}} x \exp \left(\frac{a_{6}}{a_{5}} x \exp \left(\frac{a_{7}}{a_{6}} x \tau\right)\right)\right)\right)\right)\right]=0.083 \\
& \left(m_{6}^{*}=0.394\right) .
\end{aligned}
$$

We observe two subsequences, with odd and even numbers, probably embracing the correct result from below and above, respectively. We can suspect that they both define a stable quasifixed point corresponding to a focus. In order to locate it with maximal possible precision, we can impose a minimal difference condition on the points, belonging one to the "odd" and another to "even" subsequences, with the smallest absolute values of multipliers. On the other hand, it is interesting to compare the values that can be obtained from the minimal-difference condition imposed on two starting terms of the renormalized sequence with those obtained from two last terms. E.g., from the condition $\min _{\tau}\left|\chi_{3}^{*}(\tau)-\chi_{2}^{*}(\tau)\right|$, we obtain the value of the control parameter $\tau=0.56$. Correspondingly, $\chi_{3}^{*}(\tau, S=1)=0.088$. We obtain thus, $0.087 \leq \chi^{*}(\tau, S=1) \leq 0.088$, with the lower bound following from the analysis of two last terms and of two approximants with minimal multipliers. Our estimate should be compared to the result of Ref. [76], $0.095 \pm 0.002$, obtained by Padé-summation.

The case of $S=\frac{1}{2}$ may be treated by analogy with the case of $S=1$. Following literally the same steps as above, we obtain $0.062 \leq \chi^{*}\left(\tau, S=\frac{1}{2}\right) \leq 0.064$, being close to the result of Padé-summation, $0.065 \pm 0.003$ [75].

The ground state energy $E$ of the two-dimensional Heisenberg antiferromagnet may be presented in the form of an expansion $[75,76]$ :

$$
\begin{gathered}
2 E \simeq-4+a_{2} x^{2}+a_{4} x^{4}+a_{6} x^{6}+a_{8} x^{8} \quad(x \rightarrow 0), \\
a_{2}=-\frac{4}{3}, \quad a_{4}=0.0064, \quad a_{6}=-2 \times 0.00632628, \quad a_{8}=-2 \times 0.0030085 \quad\left(S=\frac{1}{2}\right) ; \\
a_{2}=-0.571428, \quad a_{4}=-0.0504597, \quad a_{6}=-0.0144762, \quad a_{8}=-0.00656238 \quad(S=1) .
\end{gathered}
$$

In the case of spin-1, the values of the local multipliers $m_{k}(s)$ are increasing with increasing index $k$. In order to choose the starting term in (110), we compared the values of $m_{1}(\infty)=1-a_{2} / 4=1.143$ with $m_{2}(\infty)=1+a_{4} / a_{2}=1.088$. I.e., an approximation cascade will have a more stable beginning if it starts from the second term in (110). Thus, we come to the exponential approximants

$$
2 E_{2}^{*}=-4+a_{2} x^{2} \exp \left(\frac{a_{4}}{a_{2}} x^{2} \tau\right)
$$




$$
2 E_{3}^{*}=-4+a_{2} x^{2} \exp \left[\frac{a_{4}}{a_{2}} x^{2} \exp \left(\frac{a_{6}}{a_{4}} x^{2} \tau\right)\right] .
$$

From the minimal-difference condition $\min _{\tau}\left|E_{3}^{*}(\tau)-E_{2}^{*}(\tau)\right|$ we find $\tau=1.568$ and $E_{3}^{*}(\tau, S=1)=-2.328$, close to the result of Padé-summation, $E=-2.327 \pm 0.001[76]$.

In the case of spin-1/2, the same approach is applicable, and we obtain $\tau=0.457$ and $4 E_{3}^{*}\left(\tau, S=\frac{1}{2}\right)=-2.665$. Padé-summation gives in this case, $4 E=-2.6785 \pm 0.001$ [75].

Pass now to considering magnetization $M$. In the case of spin- $\frac{1}{2}$ it is more convenient to present $M$ in the form of a series in the parameter $\delta$, related to $x$ by the equation

$$
1-\delta=\left(1-x^{2}\right)^{1 / 2} .
$$

Then [75],

$$
\begin{gathered}
2 M \simeq 1+a_{1} \delta+a_{2} \delta^{2}+a_{3} \delta^{3}+a_{4} \delta^{4}+a_{5} \delta^{5} \quad(\delta \rightarrow 0), \\
a_{1}=-\frac{4}{9}, \quad a_{2}=0.08, \quad a_{3}=-0.009319, \quad a_{4}=-0.4642, \quad a_{5}=0.08257 .
\end{gathered}
$$

The local multipliers $m_{k}$ behave quite irregularly, reflecting the behavior of the coefficients. In this situation we resort to an aposteriori analysis of the sequences of exponential approximants and corresponding aposteriori multipliers, whose values will be given in brackets. For the sequence including the constant term we observe a recurrent behavior, signaling an emergence of a limiting cycle:

$$
\begin{gathered}
2 M_{1}^{*}=\exp \left(a_{1} \delta\right)=0.641 \quad\left(m_{1}^{*}=1\right), \\
2 M_{2}^{*}=\exp \left[a_{1} \delta \exp \left(\frac{a_{2}}{a_{1}} \delta\right)\right]=0.69 \quad\left(m_{2}^{*}=0.737\right), \\
2 M_{3}^{*}=\exp \left[a_{1} \delta \exp \left(\frac{a_{2}}{a_{1}} \delta \exp \left(\frac{a_{3}}{a_{2}} \delta\right)\right)\right]=0.685 \quad\left(m_{3}^{*}=0.781\right), \\
2 M_{4}^{*}=\exp \left[a_{1} \delta \exp \left(\frac{a_{2}}{a_{1}} \delta \exp \left(\frac{a_{3}}{a_{2}} \delta \exp \left(\frac{a_{4}}{a_{3}} \delta\right)\right)\right)\right]=0.641 \quad\left(m_{4}^{*}=1\right), \\
2 M_{5}^{*}=\exp \left[a_{1} \delta \exp \left(\frac{a_{2}}{a_{1}} \delta \exp \left(\frac{a_{3}}{a_{2}} \delta \exp \left(\frac{a_{4}}{a_{3}} \delta \exp \left(\frac{a_{5}}{a_{4}} \delta\right)\right)\right)\right)\right]=0.679 \quad\left(m_{5}^{*}=0.808\right) .
\end{gathered}
$$

We construct also a different sequence of exponential approximants, not including into the renormalization procedure the constant term:

$$
\begin{gathered}
2 M_{2}^{*}=1+a_{1} \delta \exp \left(\frac{a_{2}}{a_{1}} \delta\right)=0.629 \quad\left(m_{1}^{*}=1\right), \\
2 M_{3}^{*}=1+a_{1} \delta \exp \left[\frac{a_{2}}{a_{1}} \delta \exp \left(\frac{a_{3}}{a_{2}} \delta\right)\right]=0.621 \quad\left(m_{2}^{*}=0.802\right), \\
2 M_{4}^{*}=1+a_{1} \delta \exp \left[\frac{a_{2}}{a_{1}} \delta \exp \left(\frac{a_{3}}{a_{2}} \delta \exp \left(\frac{a_{4}}{a_{3}} \delta\right)\right)\right]=0.556 \quad\left(m_{3}^{*}=5.149 \times 10^{-6}\right), \\
2 M_{5}^{*}=1+a_{1} \delta \exp \left(\frac{a_{2}}{a_{1}} \delta \exp \left(\frac{a_{3}}{a_{2}} \delta \exp \left(\frac{a_{4}}{a_{3}} \delta \exp \left(\frac{a_{5}}{a_{4}} \delta\right)\right)\right)\right)=0.613 \quad\left(m_{4}^{*}=0.722\right) .
\end{gathered}
$$

The last value agrees well with the result of Ref.[75], $2 M=0.605 \pm 0.015$. 
In the case of spin-1, the following expansion in powers of $\delta$ can be obtained [76]:

$$
\begin{gathered}
M \simeq 1+a_{2} \delta^{2}+a_{3} \delta^{3}+a_{4} \delta^{4}+a_{5} \delta^{5} \quad(\delta \rightarrow 0), \\
a_{2}=-0.326528, \quad a_{3}=0.326528, \quad a_{4}=-0.73216, \quad a_{5}=1.3001056 .
\end{gathered}
$$

The values of local multipliers $m_{k}$, in this case, suggest that inclusion into consideration of the last term destabilizes the trajectory. In this situation we again resort to the aposteriori analysis. The following sequence of exponential approximants can be readily written down, with the corresponding multipliers, calculated at $\tau=1$, shown in brackets:

$$
\begin{gathered}
M_{2}^{*}=\exp \left(a_{2} \delta^{2} \tau\right)=0.721 \quad\left(m_{1}^{*}=1\right), \\
M_{3}^{*}=\exp \left[a_{2} \delta^{2} \exp \left(\frac{a_{3}}{a_{2}} \delta \tau\right)\right]=0.887 \quad\left(m_{2}^{*}=0.206\right) \\
M_{4}^{*}=\exp \left[a_{2} \delta^{2} \exp \left(\frac{a_{3}}{a_{2}} \delta \exp \left(\frac{a_{4}}{a_{3}} \delta \tau\right)\right)\right]=0.746 \quad\left(m_{3}^{*}=0.931\right), \\
M_{5}^{*}=\exp \left[a_{2} \delta^{2} \exp \left(\frac{a_{3}}{a_{2}} \delta \exp \left(\frac{a_{4}}{a_{3}} \delta \exp \left(\frac{a_{5}}{a_{4}} \delta \tau\right)\right)\right)\right]=0.848 \quad\left(m_{4}^{*}=0.33\right) .
\end{gathered}
$$

We observe two subsequences, with odd and even numbers, probably embracing the correct result from below and above, respectively. We can suspect that they both define a stable quasifixed point, corresponding to a focus. In order to locate it with the maximal possible precision, we impose the minimal difference condition on two points, belonging to two different subsequences with the smallest values of multipliers, i.e., $\min _{\tau}\left|M_{4}^{*}(\tau)-M_{3}^{*}(\tau)\right|$. From this condition we determine $\tau=0.404$ and $M_{4}^{*}(\tau, S=1)=0.804$, in agreement with the estimate $M=0.81 \pm 0.01$ [76].

The expansions for the frequency moments, $\rho_{1}$ and $\rho_{2}$, of the intensity of light scattering on the spin-pair excitations, are available for $S=1[76]$,

$$
\begin{gathered}
2 \rho_{1} \simeq a_{0}+a_{2} x^{2}+a_{3} x^{3}+a_{4} x^{4}+a_{5} x^{5} \quad(x \rightarrow 0), \\
a_{0}=14, \quad a_{2}=0.530612, \quad a_{3}=-0.141138, \quad a_{4}=0.033837, \quad a_{5}=0.0171678 ; \\
4 \rho_{2} \simeq a_{0}+a_{2} x^{2}+a_{3} x^{3}+a_{4} x^{4}+a_{5} x^{5} \quad(x \rightarrow 0), \\
a_{0}=196, \quad a_{2}=17.469, \quad a_{3}=-5.33453, \quad a_{4}=3.32992, \quad a_{5}=0.626767 .
\end{gathered}
$$

Consider the case of $\rho_{1}$. Comparing the local multipliers, as $s \rightarrow \infty, m_{1}(\infty)=1+a_{2} / a_{0}=1.038$ and $m_{2}(\infty)=$ $1+a_{3} / a_{2}=0.734$, we conclude that the constant term should not be included into the renormalization procedure. Then, at $\tau=1$ and $x=1$, the following values of the exponential approximants can be calculated:

$$
\begin{gathered}
2\left(\rho_{1}^{*}\right)_{2}=a_{0}+a_{2} x^{2} \exp \left(\frac{a_{3}}{a_{2}} x \tau\right)=7.203 \quad\left(m_{1}^{*}=1\right) \\
2\left(\rho_{1}^{*}\right)_{3}=a_{0}+a_{2} x^{2} \exp \left(\frac{a_{3}}{a_{2}} x \exp \left(\frac{a_{4}}{a_{3}} x \tau\right)\right)=7.215 \quad\left(m_{2}^{*}=0.833\right), \\
2\left(\rho_{1}^{*}\right)_{4}=a_{0}+a_{2} x^{2} \exp \left(\frac{a_{3}}{a_{2}} x \exp \left(\frac{a_{4}}{a_{3}} x \exp \left(\frac{a_{5}}{a_{4}} x \tau\right)\right)\right)=7.222 \quad\left(m_{3}^{*}=0.585\right) .
\end{gathered}
$$

We observe a smoothly behaving sequence of multipliers. From the minimal difference condition $\min _{\tau}\left|\left(\rho_{1}^{*}\right)_{4}-\left(\rho_{1}^{*}\right)_{3}\right|$, we find $\tau=1.59325$ and $\left(\rho_{1}^{*}\right)_{4}=7.221$, in agreement with $\rho_{1}=7.22 \pm 0.02$, quoted in Ref. [76]. Identical analysis leads to the value $\left(\rho_{2}^{*}\right)_{4}=52.804(\tau=1.27)$, again in agreement with $\rho_{2}=53.0 \pm 0.3$ from Ref.[76]. The value of the parameter $R=\left(\sqrt{\rho_{2}-\rho_{1}^{2}}\right) / \rho_{1}$ is equal to 0.113 , close to $0.12 \pm 0.3$ from [76]. 


\section{CRITICAL PHENOMENA}

\section{A. Martinelli-Parisi $\epsilon-$ Expansion}

Martinelli and Parisi suggested an interesting way to control the position-space renormalization group calculations [77], connecting the approximate Migdal-Kadanoff transformation with the exact theory by means of the control shift parameter $\epsilon$, equal to zero for Migdal-Kadanoff approximation and equal to one for the exact renormalization transformation. This approach generates the expansions in powers of $\epsilon$, considered as a small parameter, around the Migdal-Kadanoff results. Finally, in order to reach the "exact" solution, one should set $\epsilon=1$. The results can be further improved by imposing the condition on zero derivative of physical quantities at $\epsilon=1[77,78]$.

The following expansion for the critical index $\nu$ of the Ising model is available [77] for the square lattice:

$$
\begin{aligned}
\nu^{-1} & \simeq w_{0}+w_{1} \epsilon+w_{2} \epsilon^{2} \quad(\epsilon \rightarrow 0), \\
w_{0} & =0.687, w_{1}=1.14, w_{2}=-1.21 .
\end{aligned}
$$

Directly for the index $\nu$, one can find

$$
\begin{gathered}
\nu \simeq a_{0}+a_{1} \epsilon+a_{2} \epsilon^{2} \quad(\epsilon \rightarrow 0), \\
a_{0}=1.456, \quad a_{1}=-2.415, \quad a_{2}=6.572 .
\end{gathered}
$$

When only two starting terms from (135) are considered, the result is definitely wrong, $\nu=-0.96$, and with three terms we get $\nu=5.61$. This shows how expansion (135) is bad. The renormalization procedure, not including into consideration the constant term, gives the exponential approximant

$$
\nu_{2}^{*}(\epsilon)=a_{0}+a_{1} \epsilon \exp \left(\frac{a_{2}}{a_{1}} \epsilon\right)=1.297 .
$$

This result, is, probably, too large and it does change much, to the value 0.568 , when the condition on zero derivative is imposed. Then, in order to extend the validity of the expansion (135), let us add to it a negative trial term $-\left|a_{3}\right| \epsilon^{3}$. The following renormalized expression can be written:

$$
\nu_{3}^{*}\left(\epsilon, a_{3}\right)=a_{0}+a_{1} \epsilon \exp \left(\frac{a_{2}}{a_{1}} \epsilon \frac{1}{1+\frac{\left|a_{3}\right|}{a_{2}} \epsilon}\right) .
$$

From the condition equivalent to (45),

$$
\frac{\partial \nu_{3}^{*}\left(\epsilon, a_{3}\right)}{\partial \epsilon}=0
$$

at $\epsilon=1$, we find $a_{3}=4.268$ and $\nu_{3}^{*}(\epsilon=1)=0.992$, in excellent agreement with the exact result, $\nu=1$. Our estimate is much better than the result of Padé-summation $\nu=0.945$, quoted in [77].

For the critical temperature $T_{c}$ the following expansion was obtained [77]:

$$
\begin{gathered}
T_{c}^{-1} \simeq b_{0}+b_{1} \epsilon+b_{2} \epsilon^{2} \quad(\epsilon \rightarrow 0), \\
b_{0}=0.4359, \quad b_{1}=0.024, \quad b_{2}=-0.109 .
\end{gathered}
$$

This gives for $T_{c}$

$$
\begin{aligned}
& T_{c} \simeq a_{0}+a_{1} \epsilon+a_{2} \epsilon^{2} \quad(\epsilon \rightarrow 0), \\
& a_{0}=2.294, \quad a_{1}=-0.126, \quad a_{2}=0.581 .
\end{aligned}
$$

Two exponential approximants can be written, 


$$
\begin{gathered}
\left(T_{c}^{*}\right)_{1}=a_{0} \exp \left(\frac{a_{1}}{a_{0}} \epsilon \tau\right), \\
\left(T_{c}^{*}\right)_{2}=a_{0} \exp \left[\frac{a_{1}}{a_{0}} \epsilon \exp \left(\frac{a_{2}}{a_{1}} \epsilon \tau\right)\right] .
\end{gathered}
$$

From the minimal difference condition, at $\epsilon=1$, we find $\tau=0.278$ and $\left(T_{c}^{*}\right)_{2}=2.259$. The result does not change much (to the value $2.248, \tau=0.217$ ) when the condition on zero derivative of $\left(T_{c}^{*}\right)_{2}$ is imposed. Let us add to the expansion (139) one more term $-\left|a_{3}\right| \epsilon^{3}$, so that the following approximant can be written:

$$
\left(T_{c}^{*}\right)_{3}=a_{0} \exp \left[\frac{a_{1}}{a_{0}} \epsilon \exp \left(\frac{a_{2}}{a_{1}} \epsilon \frac{1}{1+\frac{\left|a_{3}\right|}{a_{2}} \epsilon}\right)\right],
$$

an determine $a_{3}$ from the condition on zero derivative at $\epsilon=1$. Then, $\left|a_{3}\right|=0.664$ and $\left(T_{c}^{*}\right)_{3}=2.279$. Padé approximants in this case give a close result, $T_{c}=2.275$ [77]. This is to be compared with the exact $T_{c}=2.269$.

On a triangular lattice, the following expression for $\nu$ was obtained [78] :

$$
\begin{gathered}
2^{1 / \nu} \simeq a_{0}+a_{1} \epsilon+a_{2} \epsilon^{2} \quad(\epsilon \rightarrow 0), \\
a_{0}=1.6786, \quad a_{1}=0.5344, \quad a_{2}=-0.3952 .
\end{gathered}
$$

In this case, the exponential approximant

$$
\left(2^{1 / \nu}\right)^{*}=a_{0} \exp \left[\frac{a_{1}}{a_{0}} \epsilon \exp \left(\frac{a_{2}}{a_{1}} \epsilon\right)\right],
$$

leads to $\nu^{*}=1.035$, which is a much better value than 1.161 obtained in Ref.[78] directly from (143). It is possible to improve our estimate performing the last step of the self-similar bootstrap along the most stable available trajectory, with the stabilizer $s$ corresponding to zero value of the local multiplier $m=1+a_{2}(1+s) / a_{1} s$. This yields

$$
\left(2^{1 / \nu}\right)^{*}=a_{0} \exp \left[\frac{a_{1}}{a_{0}} \epsilon\left(\frac{s}{s-\frac{a_{2}}{a_{1}} \epsilon \tau}\right)^{s}\right], \quad s=-\frac{a_{2} \epsilon}{a_{1}+a_{2} \epsilon}=2.86 .
$$

Then, $\nu^{*}=1.015$ for $\tau=1$. The derivative of $\nu^{*}$ is equal to 0.035 . At the point $\tau=1.152$, the derivative goes to zero, and our estimate changes slightly to $\nu^{*}=1.036$.

For the inverse critical temperature on a triangular lattice [78], we have

$$
\begin{aligned}
& T_{c}^{-1} \simeq b_{0}+b_{1} \epsilon+b_{2} \epsilon^{2} \quad(\epsilon \rightarrow 0), \\
b_{0}= & 0.3047, \quad b_{1}=-0.0976, \quad b_{2}=0.0501 .
\end{aligned}
$$

For the critical temperature we obtain

$$
\begin{gathered}
T_{c} \simeq a_{0}+a_{1} \epsilon+a_{2} \epsilon^{2} \quad(\epsilon \rightarrow 0), \\
a_{0}=3.282, \quad a_{1}=1.051, \quad a_{2}=-0.203 .
\end{gathered}
$$

The following exponential approximant is favored from the viewpoint of local multipliers:

$$
T_{c}^{*}=a_{0}+a_{1} \epsilon \exp \left(\frac{a_{2}}{a_{1}} \epsilon \tau\right) .
$$

The derivative of (148) is quite large at $\tau=1$, so we resort to the condition

$$
\frac{\partial T_{c}^{*}}{\partial \epsilon}=0
$$


and at $\epsilon=1$ we find $\tau=-a_{2} / a_{1}=5.177$, and

$$
T_{c}^{*}=a_{0}+a_{1} e^{-1}=3.669,
$$

deviating from the exact value 3.642 with the percentage error $0.741 \%$, while the result 3.888 , following from expansion (146) and quoted in [78], gives the error $6.755 \%$.

For the first coefficient of the beta function $\beta_{1}$ of the two-dimensional non-linear sigma-model the following expansion was obtained [79]:

$$
\begin{aligned}
\beta_{1} \simeq \frac{\sqrt{3}}{\ln (2)}\left(a_{0}+a_{1} \epsilon+a_{2} \epsilon^{2}\right) & (\epsilon \rightarrow 0), \\
a_{0}=-\frac{1}{24}, \quad a_{2}=\frac{3}{64}, & a_{3}=-\frac{69}{86} .
\end{aligned}
$$

The exponential approximant

$$
\beta_{1}^{*}=\frac{\sqrt{3}}{\ln (2)}\left(a_{0}+a_{1} \epsilon \exp \left(\frac{a_{2}}{a_{1}} \epsilon\right)\right)=-0.081
$$

is in good agreement with the exact result $\beta_{1}=-\frac{1}{4 \pi}=-0.08$ [79]. The derivative of $\beta_{1}^{*}$ is small and equals to 0.006 , so that we can safely stop at this point.

\section{B. Localization Length}

The critical exponent $\nu$ describing the divergence of the localization length in the vicinity of the Anderson transition from the insulating to the conducting phase,

$$
l \sim\left(E_{c}-E\right)^{-\nu},
$$

as the energy $E$ of an electron approaches the mobility edge $E_{c}$, can be presented in the form of $(2+\epsilon)$-expansion, or $(d-2)$-expansion, where $d$ is the dimensionality of space [80],

$$
\begin{array}{r}
\nu \simeq \frac{1}{\epsilon}+b \epsilon^{2}+c \epsilon^{3} \quad(\epsilon \rightarrow 0), \\
b=-\frac{9}{4} \zeta(3), \quad c=\frac{27}{16} \zeta(4) .
\end{array}
$$

The result given by the starting two terms is wrong, $\nu=-1.705$, while it is known that $\nu \geq 2 / 3$ in the threedimensional case $(\epsilon=1)$ [81]. The third term slightly improves the situation, but the result remains small, $\nu=0.122$. Renormalizing the starting two terms yields the exponential approximant

$$
\nu^{*}(\epsilon, \tau)=\frac{1}{\epsilon} \exp \left(b \epsilon^{3} \tau\right) .
$$

Let us impose the condition

$$
\frac{\partial \nu^{*}(\epsilon, \tau)}{\partial \epsilon}=0
$$

discussed above in Section IV. Then, the control function $\tau(\epsilon)$ is found as follows:

$$
\tau(\epsilon)=\frac{1}{3 b \epsilon^{3}} .
$$

At $\epsilon=1$, we obtain $\tau=-0.123246$, corresponding to the necessity to move backwards, from the wrong point of the approximation cascade. Our estimate for the critical index $\nu^{*}=\exp \left(\frac{1}{3}\right)=1.39561$ better agrees with the numerical result $1.3[82]$ than the Padé-Borel estimate 0.730 quoted in Ref.[80]. 


\section{Amplitude Ratios}

Consider the $3 d$ Ising model. Different amplitude ratios are available in the form of the Wilson $\epsilon$-expansion $(\epsilon=4-d)$ around the dimensionality four [83]. For the ratio $C^{+} / C^{-}$, related to the magnetic susceptibility in zero field, the following expression is available:

$$
\begin{gathered}
\frac{C^{+}}{C^{-}} \simeq 2^{\gamma}\left[1+a_{1} \epsilon+a_{2} \epsilon^{2}+a_{3} \epsilon^{3}\right] \quad(\epsilon \rightarrow 0), \\
a_{1}=\frac{1}{2}, \quad a_{2}=\frac{25}{108}, \quad a_{3}=\frac{1}{24} \lambda+\frac{1}{36} \zeta(3)+\frac{1159}{11664}, \quad \lambda=1.171953 .
\end{gathered}
$$

In order to improve the stability of the procedure, let us invert $C^{+} / C^{-}$and study

$$
\begin{gathered}
\left(\frac{C^{+}}{C^{-}}\right)^{-1} \simeq 2^{-\gamma}\left[1+b_{1} \epsilon+b_{2} \epsilon^{2}+b_{3} \epsilon^{3}\right] \quad(\epsilon \rightarrow 0), \\
b_{1}=-\frac{1}{2}, \quad b_{2}=\frac{1}{54}, \quad b_{3}=-7.551910^{-2} .
\end{gathered}
$$

From the viewpoint of stability conditions, the following two approximants are well justified:

$$
\begin{gathered}
\left(\left(\frac{C^{+}}{C^{-}}\right)^{-1}\right)_{2}^{*}=2^{-\gamma}\left[1+b_{1} \epsilon \exp \left(\frac{b_{2}}{b_{1}} \epsilon \tau\right)\right], \\
\left(\left(\frac{C^{+}}{C^{-}}\right)^{-1}\right)_{3}^{*}=2^{-\gamma}\left[1+b_{1} \epsilon \exp \left(\frac{b_{2}}{b_{1}} \epsilon \exp \left(\frac{b_{3}}{b_{2}} \epsilon \tau\right)\right)\right],
\end{gathered}
$$

and from the minimal difference condition

$$
\min _{\tau}\left|\left(\left(\frac{C^{+}}{C^{-}}\right)^{-1}\right)_{3}^{*}-\left(\left(\frac{C^{+}}{C^{-}}\right)^{-1}\right)_{2}^{*}\right|,
$$

with the typical value of $\gamma=1.24$, we obtain $\tau=0.297$. Correspondingly, $\left(C^{+} / C^{-}\right)^{*}=4.673$. This value agrees well with the theoretical estimates and experimental data [83].

The ratio $A^{+} / A^{-}$is related to the two-point correlation function at zero momentum,

$$
\begin{gathered}
A^{+} / A^{-} \simeq 2^{\alpha-2}\left[1+\epsilon-\left|a_{2}\right| \epsilon^{2}\right] \quad(\epsilon \rightarrow 0), \\
a_{2}=\frac{43}{54}-\frac{1}{6} \lambda-\zeta(3) .
\end{gathered}
$$

Two renormalized expressions can be constructed,

$$
\begin{gathered}
\left(\frac{A^{+}}{A^{-}}\right)_{1}^{*}=2^{\alpha-2}[\exp (\epsilon \tau)], \\
\left(\frac{A^{+}}{A^{-}}\right)_{2}^{*}=2^{\alpha-2}\left[\exp \left(\epsilon \exp \left(-\left|a_{2}\right| \epsilon \tau\right)\right)\right],
\end{gathered}
$$

both exponential approximants being justified from the viewpoint of stability conditions. From the condition

$$
\min _{\tau}\left|\left(\frac{A^{+}}{A^{-}}\right)_{2}^{*}-\left(\frac{A^{+}}{A^{-}}\right)_{1}^{*}\right|
$$


with the typical value of $\alpha=0.11$, we find that $\tau=0.669$ and $\left(\frac{A^{+}}{A^{-}}\right)_{2}^{*}=0.527$, well agreeing with the data of Table 5 from Ref. [83].

For the amplitude ratios $R_{c}$ and $R_{\chi}$ (see [83]), the following expansions are available:

$$
\begin{array}{r}
R_{c} \simeq \frac{1}{9} 2^{-2 \beta-1} \epsilon\left[1+a_{1} \epsilon-\left|a_{2}\right| \epsilon^{2}\right] \quad(\epsilon \rightarrow 0), \\
a_{1}=\frac{17}{27}, \quad a_{2}=\frac{989}{2916}-\frac{4}{9} \zeta(3)-\frac{2}{3} \lambda,
\end{array}
$$

and

$$
R_{\chi} \simeq 3^{(\delta-3) / 2} 2^{\gamma+(1-\delta) / 2}\left[1+\left(\frac{1}{72}+\frac{1}{36} \lambda-\frac{1}{18} \zeta(3)\right) \epsilon^{3}\right] \quad(\epsilon \rightarrow 0) .
$$

The self-similar exponential approximants are

$$
\begin{gathered}
\left(R_{c}\right)_{1}^{*}=\frac{1}{9} 2^{-2 \beta-1} \epsilon\left[\exp \left(a_{1} \epsilon \tau\right)\right] \\
\left(R_{c}\right)_{2}^{*}=\frac{1}{9} 2^{-2 \beta-1} \epsilon\left[\exp \left(a_{1} \epsilon \exp \left(-\frac{\left|a_{2}\right|}{a_{1}} \epsilon \tau\right)\right)\right] .
\end{gathered}
$$

From the minimal difference condition $\min _{\tau}\left|\left(R_{c}\right)_{2}^{*}-\left(R_{c}\right)_{1}^{*}\right|$, with the typical value $\beta=0.325$, we obtain $\tau=0.633$ and $\left(R_{c}\right)_{2}^{*}=0.053$ agreeing well with the results quoted in Ref.[83].

The renormalized expression for $R_{\chi}$ writes

$$
R_{\chi}^{*}=3^{(\delta-3) / 2} 2^{\gamma+(1-\delta) / 2}\left[\exp \left(\frac{1}{72}+\frac{1}{36} \lambda-\frac{1}{18} \zeta(3)\right) \epsilon^{3}\right]
$$

We take $\delta=4.825$, corresponding, by virtue of the scaling relation, to a reasonable value for the critical index $\eta=0.03$. Then $R_{\chi}^{*}=1.675$, close to various estimates presented in [83].

Finally, we consider the quantity $\left|z_{0}\right|$, the universal rescaled spontaneous magnetization,

$$
\begin{aligned}
& \left|z_{0}\right| \simeq \sqrt{3} 2^{\beta}\left[1+a_{1} \epsilon+a_{2} \epsilon^{2}-\left|a_{3}\right| \epsilon^{3}\right] \quad(\epsilon \rightarrow 0), \\
& a_{1}=\frac{1}{4}, \quad a_{2}=\frac{73}{864}, \quad a_{3}=\frac{1}{24} \lambda-\frac{7}{36} \zeta(3)+\frac{5581}{93312} .
\end{aligned}
$$

The self-similar exponential approximants, justified from the viewpoint of an aposteriori stability analysis, are

$$
\begin{gathered}
\left|z_{0}\right|_{2}^{*}=\sqrt{3} 2^{\beta}\left[\exp \left(a_{1} \epsilon \exp \left(\frac{a_{2}}{a_{1}} \epsilon \tau\right)\right)\right] \\
\left|z_{0}\right|_{3}^{*}=\sqrt{3} 2^{\beta}\left[\exp \left(a_{1} \epsilon \exp \left(\frac{a_{2}}{a_{1}} \epsilon \exp \left(-\frac{\left|a_{3}\right|}{a_{2}} \epsilon \tau\right)\right)\right)\right],
\end{gathered}
$$

and from the minimal difference condition $\left.\min _{\tau}|| z_{0}\right|_{3} ^{*}-\left|z_{0}\right|_{2}^{*} \mid$ we find $\tau=0.633$ and $\left|z_{0}\right|_{3}^{*}=2.913$ agreeing well with the result of Padé-summation $2.87 \pm 0.06$ [83].

\section{Critical Indices from the Wilson $\epsilon$-Expansion}

Critical indices are usually obtained from the Wilson $\epsilon$-expansion [84,85] using some kind of a resummation procedure. As a rule, for different values of $n$, standing for the number of the order parameter components, one obtains some values not related to each other analytically. We obtain below analytical renormalized expressions for the critical indices $\nu$ and $\eta$, valid for arbitrary $n$. 
For the critical index $\eta$, the expansion is available up to the fifth order term in $\epsilon$ [85]. For convenience, we reproduce the higher-order coefficients in Appendix,

$$
\begin{gathered}
\eta \simeq a_{2}(n) \epsilon^{2}+a_{3}(n) \epsilon^{3}+a_{4}(n) \epsilon^{4}+a_{5}(n) \epsilon^{5} \quad(\epsilon \rightarrow 0), \\
a_{2}(n)=\frac{n+2}{2(n+8)^{2}}, \quad a_{3}(n)=\frac{n+2}{8(n+8)^{4}}\left(272+56 n-n^{2}\right), \quad \ldots
\end{gathered}
$$

The following exponential approximants are justified from the viewpoint of the stability conditions for local multipliers:

$$
\begin{gathered}
\eta_{4}^{*}(\epsilon, n, \tau)=a_{2}(n) \epsilon^{2}+a_{3}(n) \epsilon^{3} \exp \left(\frac{a_{4}(n)}{a_{3}(n)} \epsilon \tau\right), \\
\eta_{5}^{*}(\epsilon, n, \tau)=a_{2}(n) \epsilon^{2}+a_{3}(n) \epsilon^{3} \exp \left[\frac{a_{4}(n)}{a_{3}(n)} \epsilon \exp \left(\frac{a_{5}(n)}{a_{4}(n)} \epsilon \tau\right)\right],
\end{gathered}
$$

From the minimal difference condition $\min _{\tau}\left|\eta_{5}^{*}(\epsilon, n, \tau)-\eta_{4}^{*}(\epsilon, n, \tau)\right|$, equivalent in this case to the equation

$$
\tau=\exp \left(\frac{a_{5}(n)}{a_{4}(n)} \epsilon \tau\right)
$$

we can find the control function $\tau=\tau(n)$ at $\epsilon=1$. From formula (171), we can estimate the critical index $\eta_{5}^{*}(1, n, \tau(n)) \equiv \eta^{*}(n)$. The results of calculations are presented in the Table. They agree well with the majority of different kinds of estimates available for the critical index $\eta$.

For the critical index $\nu$, up to the fifth order in $\epsilon$ (see Appendix), one has [85]

$$
\begin{gathered}
\nu^{-1} \simeq b_{0}(n)+b_{1}(n) \epsilon+b_{2}(n) \epsilon^{2}+b_{3}(n) \epsilon^{3}+b_{4}(n) \epsilon^{4}+b_{5}(n) \epsilon^{5} \quad(\epsilon \rightarrow 0), \\
b_{0}(n)=2, \quad b_{1}(n)=-\frac{n+2}{n+8}, \quad b_{2}(n)=-\frac{(n+2)(13 n+44)}{2(n+8)^{3}}, \quad \ldots
\end{gathered}
$$

The following exponential approximants, preserving the correct limits $\nu=\frac{1}{2}$ at $n=-2$ [86-88] and $\nu=1$, as $n \rightarrow \infty$ [89], can be written, giving at $\tau=1$ and $\epsilon=1$ the following results

$$
\begin{gathered}
\left(\nu^{-1}\right)_{2}^{*}=b_{0}(n)+b_{1}(n) \epsilon \exp \left[\frac{b_{2}(n)}{b_{1}(n)} \epsilon \tau\right], \\
\nu_{2}^{*}(n=0)=0.607, \quad \nu_{2}^{*}(n=1)=0.655, \quad \nu_{2}^{*}(n=2)=0.698, \quad \nu_{2}^{*}(n=3)=0.736 . \\
\left(\nu^{-1}\right)_{3}^{*}=b_{0}(n)+b_{1}(n) \epsilon \exp \left[\frac{b_{2}(n)}{b_{1}(n)} \epsilon \exp \left(\frac{b_{3}(n)}{b_{2}(n)} \epsilon \tau\right)\right], \\
\nu_{3}^{*}(n=0)=0.579, \quad \nu_{3}^{*}(n=1)=0.616, \quad \nu_{3}^{*}(n=2)=0.651, \quad \nu_{3}^{*}(n=3)=0.683 . \\
\left(\nu^{-1}\right)_{4}^{*}=b_{0}(n)+b_{1}(n) \epsilon \exp \left[\frac{b_{2}(n)}{b_{1}(n)} \epsilon \exp \left(\frac{b_{3}(n)}{b_{2}(n)} \epsilon \exp \left(\frac{b_{4}(n)}{b_{3}(n)} \epsilon \tau\right)\right)\right], \\
\nu_{4}^{*}(n=0)=0.603, \quad \nu_{4}^{*}(n=1)=0.649, \quad \nu_{4}^{*}(n=2)=0.692, \quad \nu_{4}^{*}(n=3)=0.729 . \\
\left(\nu^{-1}\right)_{5}^{*}=b_{0}(n)+b_{1}(n) \epsilon \exp \left[\frac{b_{2}(n)}{b_{1}(n)} \epsilon \exp \left(\frac{b_{3}(n)}{b_{2}(n)} \epsilon \exp \left(\frac{b_{4}(n)}{b_{3}(n)} \epsilon \exp \left(\frac{b_{5}(n)}{b_{4}(n)} \epsilon \tau\right)\right)\right] .\right. \\
\nu_{5}^{*}(n=0)=0.58, \quad \nu_{5}^{*}(n=1)=0.618, \quad \nu_{5}^{*}(n=2)=0.654, \quad \nu_{5}^{*}(n=3)=0.688 .
\end{gathered}
$$


Two sequences, with odd and even numbers, are clearly seen. The last two approximants give the closest values. From the minimal difference condition $\min _{\tau}\left|\left(\nu^{-1}\right)_{5}^{*}-\left(\nu^{-1}\right)_{4}^{*}\right|$, which simply reduces to the equation

$$
\tau=\exp \left(\frac{b_{5}(n)}{b_{4}(n)} \epsilon \tau\right)
$$

one can easily find the control function $\tau=\tau(n)$, and, finally, calculate the critical index, $\nu^{*}(n)=\left(\left(\nu^{-1}\right)_{5}^{*}\right)^{-1}$.

For the critical exponent $\omega(\epsilon)=2 B_{\epsilon}^{\prime}$, calculated in the renormalized infrared-stable fixed point, the following expansion is available [85]:

$$
\begin{gathered}
\omega(\epsilon) \simeq \epsilon+c_{2}(n) \epsilon^{2}+c_{3}(n) \epsilon^{3} \quad(\epsilon \rightarrow 0), \\
c_{2}(n)=-\frac{9 n+42}{(n+8)^{2}}, \\
c_{3}(n)=\frac{1}{4(n+8)^{4}}\left[33 n^{3}+538 n^{2}+4288 n+9658+\zeta(3)(n+8) 96(5 n+22)\right] .
\end{gathered}
$$

We limit here the discussion by the third-order terms, since the higher order terms grow impetuously, jeopardizing the fulfillment of the stability conditions. The following exponential approximants are available being well justified from the viewpoint of stability,

$$
\begin{gathered}
\omega_{2}^{*}(\epsilon, n, \tau)=\epsilon \exp \left(c_{2}(n) \epsilon \tau\right) \\
\omega_{3}^{*}(\epsilon, n, \tau)=\epsilon \exp \left[c_{2}(n) \epsilon \exp \left(\frac{c_{3}(n)}{c_{2}(n)} \epsilon \tau\right)\right],
\end{gathered}
$$

and from the minimal difference condition

$$
\tau=\exp \left(\frac{c_{3}(n)}{c_{2}(n)} \epsilon \tau\right),
$$

one can find the control function $\tau=\tau(n)$ at $\epsilon=1$. We obtain the following values of the index in the physically interesting region:

$$
\omega^{*}(n=0)=0.788, \quad \omega^{*}(n=1)=0.788, \quad \omega^{*}(n=2)=0.791, \quad \omega^{*}(n=3)=0.794 .
$$

The limiting values, $\omega^{*}(n=-2) \approx 0.8, \omega^{*}(n \rightarrow \infty) \approx 1$, sound reasonable. The dynamical critical index $\theta=\omega \nu$ can be estimated using (176) and (179). The results for the critical indices $\eta$ and $\nu$, presented in the Table, well agree with other theoretical estimates and available experimental data [90-92]. Let us stress again, that at $n=-2$ and $n \rightarrow \infty$, we obtain the exact results.

\section{E. Critical Indices from the Field Theory Expansion}

Field-theory approach in the theory of critical phenomena is, usually, very accurate [92,93]. In Ref [13] we analyzed the expansions in powers of the interaction constant $g$ ( $g$-expansion) for some critical indices from the viewpoint of the limiting cases $n=-2, n \rightarrow \infty$, and found by a direct inspection of the expressions for $\eta$ and $\gamma$ from [92], that the $n \rightarrow \infty$ limit, corresponding to the spherical model [89], is obeyed rigorously if $g=1$, i.e. $\eta=0$, $\gamma=2$, and the $n=-2$ limit, corresponding to the gaussian polymer [86-88], is obeyed with a very high accuracy for arbitrary $g$, i.e. $\eta \approx 0, \gamma \approx 1$.

The standard approach [93] uses, for computing the renormalized infrared stable fixed point $g^{*}$ of the beta-function $B_{1}(g)$, a complicated Borel summation technique. Then critical indices are calculated as $\gamma\left(g^{*}\right)$ and $\eta\left(g^{*}\right)$. The results, thus, depend on the way in which the position of the fixed point is determined, although, different approaches give the results very close to each other [92]. We suggest below a simple way to minimize an uncertainty related to the 
position of the fixed point. Let us use below two different approaches to the determination of $g^{*}$. The first approach was suggested in Ref.[13]. It is based on three starting terms from the $g$-expansion from Ref.[92] (see Appendix):

$$
\begin{gathered}
B_{1}(g) \simeq-g+g^{2}+a_{3}(n) g^{3}+a_{4}(n) g^{4}+a_{5}(n) g^{5}+a_{6}(n) g^{6} \quad(g \rightarrow 0), \\
a_{3}(n)=-\frac{6.07407408 n+28.14814815}{(n+8)^{2}}, \quad \ldots
\end{gathered}
$$

For the velocity field we have

$$
v_{3}(f)=-\frac{a_{3}}{8}\left(\frac{1}{2}(1+\sqrt{1+4 f})^{3},\right.
$$

which is to be substituted into the evolution integral

$$
\int_{-g+g^{2}}^{B_{1}^{*}} \frac{d f}{v_{3}(f)}=\tau .
$$

The root $g_{1}^{*}=g_{1}^{*}(n, \tau)$ of the equation $B_{1}^{*}(g, n, \tau=1)=0$ is obtained numerically, as a function $g_{1}^{*}=g_{1}^{*}(n)$. The following values were obtained in the physically important cases:

$$
g_{1}^{*}(n=0)=1.59, \quad g_{1}^{*}(n=1)=1.559, \quad g_{1}^{*}(n=2)=1.524, \quad g_{1}^{*}(n=3)=1.491 .
$$

Such a decreasing, with $n$, dependence is characteristic to the majority of related studies. We observed also, that at $n=-2, g_{1}^{*}=1.599$ and at $n \rightarrow \infty, g_{1}^{*}=1$. The dependence of $g^{*}(n)$ in the interval $n \in(-2,0)$ is nonmonotonous, a maximum is reached at $n=-1$, where $g_{1}^{*}=1.61$. We also constructed a different beta-function $B_{2}(g)$,

$$
B_{2}(g, \tau)=-g \exp \left(-a_{3}(n) g^{2}\right)+g^{2} \exp \left(a_{4}(n) g^{2}\right)+a_{5}(n) g^{5} \exp \left(\frac{a_{6}(n)}{a_{5}(n)} g \tau\right),
$$

leading to the following increasing with $n$ values of the fixed point $g_{2}^{*}(n, \tau)$ at $\tau=1$,

$$
g_{2}^{*}(n=0)=1.163, \quad g_{2}^{*}(n=1)=1.208, \quad g_{2}^{*}(n=2)=1.249, \quad g_{2}^{*}(n=3)=1.285,
$$

possessing a maximum at $n=7$. Such an increasing dependence better corresponds to the known decrease of the critical temperature $T_{c}$, from the Ising $(n=1)$ to Heisenberg $(n=3)$ model [72], since $T_{c} \sim g^{-1}$ [94]. We attempt now to minimize uncertainty, connected to the way of determining the fixed point. Imposing the minimal difference condition

$$
\min _{\tau}\left|g_{2}^{*}(n, \tau)-g_{1}^{*}(n, \tau)\right|,
$$

we obtain the control function $\tau=\tau(n)$, and the following values for the optimized zero of the beta-function

$$
g^{*}(n=0)=1.311, \quad g^{*}(n=1)=1.334, \quad g^{*}(n=2)=1.352, \quad g^{*}(n=3)=1.365 .
$$

These values increase till $n=5$, when $g^{*}(n=5)=1.372$, and then decrease till $g^{*}=1$, as $n \rightarrow \infty$.

For the critical index $\eta$, we keep all the terms available, up to the sixth order in powers of $g$ [92] (see Appendix):

$$
\begin{gathered}
\eta(g) \simeq b_{2}(n) g^{2}+b_{3}(n) g^{3}+b_{4}(n) g^{4}+b_{5}(n) g^{5}+b_{6}(n) g^{5} \quad(g \rightarrow 0), \\
b_{2}(n)=\frac{0.2962962963(n+2)}{(n+8)^{2}}, \quad b_{3}(n)=\frac{0.0246840014 n^{2}+0.246840014 n+0.3949440224}{(n+8)^{3}}, \ldots
\end{gathered}
$$

From the aposteriori stability analysis we select the fifth order approximant corresponding to the smallest multiplier:

$$
\eta_{5}^{*}(g, n)=b_{2}(n) g^{2} \exp \left[\frac{b_{3}(n)}{b_{2}(n)} g \exp \left(\frac{b_{4}(n)}{b_{3}(n)} g \exp \left(\frac{b_{5}(n)}{b_{4}(n)} g\right)\right)\right],
$$


so that

$$
\eta_{5}^{*}\left(g^{*}, n=0\right)=0.027, \quad \eta_{5}^{*}\left(g^{*}, n=1\right)=0.034, \quad \eta_{5}^{*}\left(g^{*}, n=2\right)=0.039, \quad \eta_{5}^{*}\left(g^{*}, n=3\right)=0.04 .
$$

These values agree well with those quoted in Refs. [91,92].

For the critical index $\gamma$ we write down all the terms available, in powers of $g$ [92] (see Appendix),

$$
\begin{gathered}
\gamma^{-1} \simeq 1+c_{1}(n) g+c_{2}(n) g^{2}+c_{3}(n) g^{3}+c_{4}(n) g^{4}+c_{5}(n) g^{5}+c_{6}(n) g^{6} \quad(g \rightarrow 0), \\
c_{1}(n)=-\frac{n+2}{2(n+8)}, \quad c_{2}(n)=\frac{n+2}{(n+8)^{2}}, \quad \ldots
\end{gathered}
$$

The third order exponential approximant is selected by an aposteriori analysis, since it corresponds to the smallest value of the multiplier, giving the following expression for the critical index:

$$
\left(\gamma^{-1}\right)_{3}^{*}=1+c_{1}(n) g \exp \left[\frac{c_{2}(n)}{c_{1}(n)} g \exp \left(\frac{c_{3}(n)}{c_{2}(n)} g\right)\right],
$$

so that

$$
\gamma^{*}\left(g^{*}, n=0\right)=1.164, \quad \gamma^{*}\left(g^{*}, n=1\right)=1.243, \quad \gamma^{*}\left(g^{*}, n=2\right)=1.319, \quad \gamma^{*}\left(g^{*}, n=3\right)=1.39
$$

which again agrees well with the data of Refs.[90-92].

\section{DISCUSSION}

In this paper we have developed an analytical approach for summing divergent series with arbitrary noninteger as well as integer powers. This approach is based on the novel notion of the self-similar exponential approximants. By a number of examples we show that the developed method is general and accurate. In addition, the exponential approximants have a simple analytical structure, even for quite large number of perturbative terms used, when one usually has to resort to numerical techniques. Because of its analytical nature, our method permits one to accomplish direct analysis of resulting formulas with respect to the variation of physical parameters. All examples we have analyzed are related to important physical phenomena, such as critical and crossover phenomena. We demonstrate that the method of self-similar exponential approximants provides an effective general tool for treating many different problems of statistical physics.

In conclusion, it is worth touching the following question. Assume that we are given a truncated series (1). Then, would it be possible, looking at the given series, to get some heuristic arguments, when the self-similar approximants in the form of nested exponentials should yield good results. The answer to this question is: Yes, we can made a preliminary estimates whether the nested exponentials would work well. Such a first-glance investigation can be done by analysing the apriori multiplier (42). Since the case of the nested exponentials corresponds to $s \rightarrow \infty$, then from (48), we have

$$
\lim _{s \rightarrow \infty} m_{k}(x, s)=\sum_{n=0}^{k} \frac{a_{n}}{a_{0}} x^{\alpha_{n}-\alpha_{0}}
$$

From here, we immediately notice what would be the favourable cases for the better stability of the procedure, which is related to the condition of the minimal multiplier modulus, $\left|m_{k}(x, \infty)\right|$. Such favorable cases include: (i) When $a_{n}$ decreases as $n$ increases, so that $\left|a_{n} / a_{0}\right|<1$ and $a_{n} / a_{0} \rightarrow 0$, as $n \rightarrow \infty$. If $\left|x^{\alpha_{n}-\alpha_{0}}\right|$ increases with $n$, then, to compensate this increase, $\left|a_{n} / a_{0}\right|$ must decrease sufficiently fast. (ii) When with increasing $n,\left|x^{\alpha_{n}-\alpha_{0}}\right|$ decreases. This kind of situation occurs, e.g., in the strong-coupling limit of many quantum problems, when $\alpha_{n}<0$, and $x^{\alpha_{n}-\alpha_{0}} \rightarrow 0$, as $n \rightarrow \infty$. If the decrease of $\left|x^{\alpha_{n}-\alpha_{0}}\right|$ is sufficiently fast, then $\left|a_{n} / a_{0}\right|$ may even grow with $n$. (iii) When (1) is an alternating series, that is, the coefficients $a_{n}$ change their signs with changing $n$. In this case, even if neither $\left|a_{n} / a_{0}\right|$ nor $\left|x^{\alpha_{n}-\alpha_{0}}\right|$ decrease, but nevertheless, because of alternating signs of $a_{n}$, the value $\left|m_{k}(x, \infty)\right|$ may be small.

The worst case, as is seen from (187), would be when all coefficients $a_{n}$ are of the same sign, $a_{n}$ increases with $n$, so that $a_{n} / a_{0}>1$, and in addition, when $x^{\alpha_{n}-\alpha_{0}}>1$. To explain why this case is really the worst, consider a simple illustration for series (1) truncated at second order,

$$
p_{2}(x)=a_{0}+a_{1} x+a_{2} x^{2} .
$$


The related apriori multiplier (187) is

$$
m_{2}(x, \infty)=1+\frac{a_{1}}{a_{2}} x+\frac{a_{2}}{a_{0}} x^{2} .
$$

Starting with $p_{2}(x)$, let us construct the simplest nested exponentials, not invoking control functions,

$$
f_{1}^{*}(x)=a_{0} \exp \left(\frac{a_{1}}{a_{0}} x\right), \quad f_{2}^{*}(x)=a_{0} \exp \left(\frac{a_{1}}{a_{0}} x \exp \left(\frac{a_{2}}{a_{1}} x\right)\right) .
$$

The sequence $\left\{f_{k}^{*}(x)\right\}$ has to satisfy the stability condition (50), with the aposteriori multipliers (49). In our case, $m_{1}^{*}(x)=1$ and

$$
m_{2}^{*}(x)=\left(1+\frac{a_{2}}{a_{1}} x\right) \exp \left(\frac{a_{2}}{a_{1}} x\right) \frac{f_{2}^{*}(x)}{f_{1}^{*}(x)} .
$$

Assume now that $a_{n+1}>a_{n}>0$ and $x>0$. Then $f_{n+1}^{*}(x)>f_{n}^{*}(x)$. In such a case, multiplier (190) is more than unity. Hence, the procedure is locally unstable, and we cannot trust to approximants (189). The first of them, i.e. $f_{1}^{*}(x)$, can yet give a reasonable estimate, but the second, $f_{2}^{*}(x)$, is certainly untrustable.

In this way, a quick glance at the apriori multiplier (187) gives us a feeling of whether the self-similar exponentials would produce good results. But the final conclusion of whether we did have managed to construct a convergent sequence of nested exponential approximants is to be based on the stability analysis of the renormalized multipliers (49). The relation between the latter and the apriori multipliers (187) is not direct, as can be seen even from the simplest case resulting in (188) and (190). In general, it would be more correct to say that there is no direct relation between these two types of multipliers. Therefore, there is no necessity of requiring that the apriori multipliers (187) be compulsory less than unity in their absolute values. It is sufficient to require that the final multipliers (49) satisfy the stability condition (50).

Another question that may arise is as follows. Suppose, we have met with the worst case, when we are not able to construct a convergent sequence of self-similar exponential approximants. How then should we proceed in order to define an effective limit of a divergent sequence $\left\{p_{k}(x)\right\}$ ? In such a case, there are several possibilities. First of all, since it is always $m_{1}^{*}(x)=1$, as is clear from definition (49), thence $f_{1}^{*}(x)$ may serve as an estimate for an effective limit of the sequence $\left\{p_{k}(x)\right\}$.

Second, recall that the nested exponentials are only one of admissible forms of self-similar approximants, corresponding to a particular case, when the power in the algebraic transformation (2) is assumed to tend to infinity. If the latter assumption is waved aside, we return to the radical form (10). Then, we have to define control functions $s=s_{k}(x)$ and $\tau=\tau_{k}(x)$ so that the sequence $\left\{p_{k}^{*}\left(x, s_{k}, \tau_{k}\right)\right\}$ be convergent. Equations defining these control functions, as always in the framework of the self-similar approximation theory [7-14], follow from the requirement for the corresponding approximation cascade to have a stable fixed point; the stability of a fixed point being in one-to-one correspondence with the existence of a limit which the sequence of approximations converges to $[10,11,95]$. Developing this procedure, we come to a convergent sequence $\left\{f_{k}^{*}(x)\right\}$ of self-similar approximants $f_{k}^{*}(x)=p_{k}^{*}\left(x, s_{k}(x), \tau_{k}(x)\right)$ which can give very accurate approximations for the sought function, but will not have such a nice structure as that of nested exponentials.

The self-similar exponential approximants, in addition to having a nice and convenient mathematical structure, evidently illustrate by their form the idea of self-similarity of approximations [7-9]. Thus, if we introduce a function

$$
G(x, y) \equiv x e^{y}
$$

and use the notation

$$
x_{i} \equiv b_{i} x^{\beta_{i}} ; \quad x_{0} \equiv a_{0} x^{\alpha_{0}},
$$

then the self-similar approximant (18) can be written as

$$
F_{k}(x)=G\left(x_{0}, G\left(x_{1}, \ldots G\left(x_{k-1}, x_{k}\right)\right) \ldots\right) .
$$

Finally, one more possibility of treating divergent series, when the direct construction of nested exponentials does not work, could be either by resorting to a change of variables or by invoking a transformation of the given series, so that the following application of the self-similar approximation theory would result in a convergent sequence of nested exponentials. What kind of a transformation or a change of variables is appropriate can again be decided by means of the apriori multipliers (187). 


\section{Acknowledgment}

We are grateful to E.P. Yukalova for discussions and advice. We appreciate financial support from the National Science and Technology Development Council of Brazil and from the University of Western Ontario, Canada. 


\section{Appendix}

The higher-order coefficients in the $\epsilon$-expansion for the critical index $\eta$ are [85]

$$
\begin{gathered}
a_{4}(n)=-\frac{1}{32} \frac{n+2}{(n+8)^{6}} \times \\
\times\left[5 n^{4}+230 n^{3}-1124 n^{2}-17920 n-46144+\zeta(3)(n+8) 384(5 n+22)\right] \\
a_{5}(n)=-\frac{1}{128} \frac{n+2}{(n+8)^{8}} \times \\
\times\left[\left(13 n^{6}+946 n^{5}+27620 n^{4}+121472 n^{3}-262528 n^{2}-2912768 n-5655552\right)-\right. \\
-\zeta(3)(n+8) 16\left(n^{5}+10 n^{4}+1220 n^{3}-1136 n^{2}-68672 n-171264\right)+ \\
\left.+\zeta(4)(n+8)^{3} 1152(5 n+22)-\zeta(5)(n+8)^{2} 5120\left(2 n^{2}+55 n+186\right)\right] .
\end{gathered}
$$

For the critical index $\nu$ one has

$$
\begin{gathered}
b_{3}(n)=\frac{(n+2)}{8(n+8)^{5}}\left[3 n^{3}-452 n^{2}-2672 n-5312+\zeta(3)(n+8) 96(5 n+22)\right] \\
b_{4}(n)=\frac{(n+2)}{32(n+8)^{7}}\left[3 n^{5}+398 n^{4}-12900 n^{3}-81552 n^{2}-219968 n-357120+\right. \\
+\zeta(3)(n+8) 16\left(3 n^{4}-194 n^{3}+148 n^{2}+9472 n+19488\right)+ \\
\left.+\zeta(4)(n+8)^{3} 288(5 n+22)-\zeta(5)(n+8)^{2} 1280\left(2 n^{2}+55 n+186\right)\right], \\
\times\left[3 n^{7}-1198 n^{6}-27484 n^{5}-1055344 n^{4}-5242112 n^{3}-5256704 n^{2}+6999040 n-626688-\right. \\
-\zeta(3)(n+8) 16\left(13 n^{6}-310 n^{5}+19004 n^{4}+102400 n^{3}-381536 n^{2}-2792576 n-4240640\right)- \\
-\zeta^{2}(3)(n+8)^{2} 1024\left(2 n^{4}+18 n^{3}+981 n^{2}+6994 n+11688\right)+ \\
+\zeta(4)(n+8)^{3} 48\left(3 n^{4}-194 n^{3}+148 n^{2}+9472 n+19488\right)+ \\
+\zeta(5)(n+8)^{2} 256\left(155 n^{4}+3026 n^{3}+989 n^{2}-66018 n-130608\right)- \\
\left.-\zeta(6)(n+8)^{4} 6400\left(2 n^{2}+55 n+186\right)+\zeta(7)(n+8)^{3} 56448\left(14 n^{2}+189 n+526\right)\right] .
\end{gathered}
$$

The higher-order coefficients of the field-theory expansion [92] for the $\beta$-function are

$$
a_{4}(n)=\frac{1}{(n+8)^{3}}\left(1.34894276 n^{2}+54.94037698 n+199.6404170\right),
$$




$$
\begin{gathered}
a_{5}(n)=-\frac{1}{(n+8)^{4}}\left(-0.15564589 n^{3}+35.82020378 n^{2}+602.5212305 n+1832.206732\right), \\
a_{6}(n)=\frac{1}{(n+8)^{5}} \times \\
\times\left(0.05123618 n^{4}+3.23787620 n^{3}+668.5543368 n^{2}+7819.564764 n+20770.17697\right) .
\end{gathered}
$$

For the critical index $\eta$,

$$
\begin{gathered}
b_{4}(n)=\frac{1}{(n+8)^{4}}\left(-0.0042985626 n^{3}+0.6679859202 n^{2}+4.609221057 n+6.512109933\right. \\
\begin{array}{r}
b_{5}(n)=-\frac{1}{(n+8)^{5}}\left(0.0065509222 n^{4}-0.1324510614 n^{3}+1.891139282 n^{2}+15.18809340 n+\right. \\
+21.64720643), \\
b_{6}(n)=\frac{1}{(n+8)^{6}}\left(-0.005548920 n^{5}-0.0203994485 n^{4}+3.054030987 n^{3}+64.07744656 n^{2}+\right. \\
+300.7208933 n+369.7130739) .
\end{array}
\end{gathered}
$$

For the critical index $\gamma$,

$$
\begin{gathered}
c_{3}(n)=-\frac{1}{(n+8)^{3}}\left(0.8795588926 n^{2}+6.485476868 n+9.452718166\right), \\
c_{4}(n)=\frac{1}{(n+8)^{4}}\left(-0.1283321043 n^{3}+7.966740703 n^{2}+51.84421298 n+70.79480631\right), \\
c_{5}(n)=-\frac{1}{(n+8)^{5}}\left(0.0490966058 n^{4}+4.288152493 n^{3}+108.3618219 n^{2}+537.8136105 n+\right. \\
+675.6996077), \\
c_{6}(n)=\frac{1}{(n+8)^{6}}\left(-0.0259267945 n^{5}-1.618627843 n^{4}+85.54569746 n^{3}+1538.818235 n^{2}+\right. \\
+6653.956526 n+7862.074086) .
\end{gathered}
$$

[1] G. A. Baker Jr. and P. Graves-Moris, Padé-Approximants (Cambridge Univ., Cambridge, 1996).

[2] R. Simon, Bull. Amer. Math. Soc. 24, 303 (1991).

[3] G. A. Baker Jr. , G. S. Rashbrooke, and H. E. Gilbert, Phys. Rev. A 135, 1272 (1964).

[4] J. Čižek, E. J. Weniger, P. Bracken, and V. Špirko, Phys. Rev. E 53, 2925 (1996).

[5] E. J. Weniger, Comput. Phys. Rep. 10, 189 (1989). 
[6] E. J. Weniger, Comput. Phys. 10, 496 (1989).

[7] V. I. Yukalov, Physica A 167, 833 (1990).

[8] V. I. Yukalov, J. Math. Phys. 32, 1235 (1991).

[9] V. I. Yukalov, J. Math. Phys. 33, 3994 (1992).

[10] V. I. Yukalov and E. P. Yukalova, Physica A 206, 553 (1994).

[11] V. I. Yukalov and E. P. Yukalova, Physica A 225, 336 (1996).

[12] V. I. Yukalov and S. Gluzman, Phys. Rev. Lett. 79, 333 (1997).

[13] S. Gluzman and V. I. Yukalov, Phys. Rev. E 55, 3983 (1997).

[14] V. I. Yukalov and S. Gluzman, Phys. Rev. E 55, 6552 (1997).

[15] J. Aczél, Lectures on Functional Equations and Their Applications (Academic, New York, 1966).

[16] I. G. Petrovsky, Lectures on the Theory of Ordinary Differential Equations (Nauka, Moscow, 1970).

[17] N. N. Bogolubov and D. V. Shirkov, Quantum Fields (Benjamin, London, 1983).

[18] L. Euler, Acta Acad. Petropolitanae 1, 38 (1777).

[19] R. Knoebel, Am. Math. Mon. 88, 235 (1981).

[20] C. M. Bender and J. P. Winson, J. Math. Phys. 37, 4103 (1996).

[21] B. J. West and W. Deering, Phys. Rep. 246, 1 (1994).

[22] D. Sornette and C. G. Sammins, J. Phys. I France 5, 607 (1995).

[23] D. Sornette, A. Johansen, and J. P. Bouchaud, J. Phys. I France, 6, 167 (1996).

[24] H. Saleur and D. Sornette, J. Phys. I France, 6, 327 (1996).

[25] J. Zinn-Justin, Quantum Field Theory and Critical Phenonema (Claredon, Oxford, 1996).

[26] V. I. Yukalov, Mosc. Univ. Phys. Bull. 31, 10 (1976).

[27] V. I. Yukalov, Theor. Math. Phys. 28, 652 (1976).

[28] I. Halliday and P. Suranyi, Phys. Rev. D 21, 1529 (1980).

[29] W. E. Caswell, Ann. Phys. (N.Y) 123, 153 (1979).

[30] P. M. Stevenson, Phys. Rev. D 23, 2916 (1981).

[31] J. Killingbeck, J. Phys. A 14, 1005 (1981).

[32] I. D. Feranchuk and L. I. Komarov, Phys. Lett. A 88, 211 (1982).

[33] E. P. Yukalova and V. I. Yukalov, Phys. Lett. A 175, 27 (1993).

[34] A. Okopińska, Phys. Lett. B 375, 213 (1996).

[35] E. J. Weniger, Ann. Phys. (N.Y.) 246, 133 (1996).

[36] A. Okopińska, Int. J. Mod. Phys. A 12, 585 (1997).

[37] A. Duncan and H. F. Jones, Phys. Rev. D 47, 2560 (1993).

[38] C. M. Bender, A. Duncan, and H. F. Jones, Phys. Rev. D 49, 4219 (1994).

[39] R. Guida, K. Konishi, and H. Suzuki, Ann. Phys. (N.Y) 241, 152 (1995).

[40] R. Guida, K. Konishi, and H. Suzuki, Ann. Phys. (N.Y.) 249, 109 (1996).

[41] V. I. Yukalov and E. P. Yukalova, Can. J. Phys. 71, 537 (1993).

[42] E. H. Lieb and W. Liniger, Phys. Rev. 130, 1605 (1963).

[43] M. Girardeau, J. Math. Phys. 1, 516 (1960).

[44] K. Yamada, Prog. Theor. Phys. 62, 354 (1979).

[45] E. J. Weniger, Phys. Rev. Lett. 77, 2859 (1996).

[46] D. Horn and M. Weinstein, Phys. Rev. D 30, 1256 (1984).

[47] L. Hulthen, Arkiv. Mat. Astron. Fysik A 26, N11 (1938).

[48] A. C. Irving, T. E. Preece, and C. J. Hamer, Nucl. Phys. B 270 [FS16], 537 (1986).

[49] C. G. Callan, R. F. Dashen, and D. J. Gross, Phys. Rev. D 20 , 3279 (1979).

[50] J. B. Kogut, Phys. Rep. 67, 67 (1980).

[51] J. Kondo, Prog. Theor. Phys. 32, 37 (1964).

[52] A. A. Abrikosov and A. A. Migdal, J. Low. Temp. Phys. 3, 519 (1970).

[53] M. Fowler and A. Zawadowski, Solid State. Commun. 9, 471 (1971).

[54] Y. A. Izumov and Y. N. Skryabin, Statistical Mechanics of Magnetically Ordered Systems (Consultants Bureau, New York, 1988).

[55] N. Andrei, Phys. Rev. Lett. 45, 379 (1980).

[56] P. B. Wiegmann, JETP Lett. 31, 364 (1980).

[57] K. G. Wilson, Rev. Mod. Phys. 47, 773 (1975).

[58] D. L. Cox and M. Jarrell, J. Phys. Condens. Matter 8, 9825 (1996).

[59] A. O. Gogolin, Z. Phys. B 92, 55 (1993).

[60] J. M. Ziman, Models of Disorder (Cambridge University Press, Cambridge, 1979).

[61] J. S. Rowlinson, in Many-Body Problems (W. A. Benjamin, New York, 1969), p.93.

[62] S. Shinomoto, J. Stat. Phys. 32, 105 (1983).

[63] C. P. Bokis and M. D. Donohue, J. Phys. Chem. 99, 12655 (1995).

[64] A. R. Altenberger and J. S. Dahler, Phys. Rev. E 54, 6242 (1996). 
[65] C. Keller, M. de Liano, S. Z. Ren, M. A. Solis and G. A. Baker, Jr, Ann. Phys. (N.Y), 251, 64 (1996).

[66] F. London, Superfluids, Vol.II, (Dover, New York, 1964), p.30.

[67] H. Yamakawa, Modern Theory of Polymer Solutions (Harper and Row, New York, 1971).

[68] M. Muthukumar and B. G. Nickel, J. Chem. Phys. 80, 5893 (1984).

[69] A. J. Barrett and C. Domb, J. Stat. Phys. 77, 491 (1994).

[70] M. Muthukumar and B. G. Nickel, J. Chem. Phys. 86, 460 (1987).

[71] H. E. Stanley, Introduction to Phase Transitions and Critical Phenomena (Clarendon, Oxford, 1982).

[72] C. Domb, in Magnetism, Vol. II, Part A, edited by G. T. Rado and H. Suhl (Academic Press. New York, 1965$)$, p.2.

[73] N. H. March, and M. Parinello, Collective Effects in Solids and Liquids (Adam Higler, Bristol, 1982).

[74] D. M. Burley, Phil. Mag. 5, 909 (1960).

[75] R. R. P. Singh, Phys. Rev. B 39, 9760 (1989).

[76] R. R. P. Singh, Phys. Rev. B 41, 4873 (1990).

[77] G. Martinelli and G. Parisi, Nucl. Phys. B 180, 201 (1981).

[78] S. Caracciollo, Nucl. Phys. B 180 [FS2], 405 (1981).

[79] S. Belforte and P. Menotti, Nucl. Phys. B 205 [FS5], 325 (1982).

[80] S. Hikami, Prog. Theor. Phys. Suppl. 107, 213 (1992).

[81] J. T. Chayes, L. Chayes, D. S. Fisher, and T. Spencer, Phys. Rev. Lett. 57, 1383 (1986).

[82] B. Kramer, K. Broderix, A. Mackinnon, and M. Shreiber, Physica A 167, 163 (1990).

[83] R. Guida and J. Zinn-Justin, Nucl. Phys. B 489 [FS], 626 (1997).

[84] K. G. Wilson and J. Kogut, Phys. Rep. 12, 75 (1974).

[85] H. Kleinert, J. Neu, V. Schulte-Frohlinde, K. G. Chetyrkin, and S. A. Larin, Phys. Lett. B 272, 39 (1991).

[86] D. Stauffer, Bull. Am. Phys. Soc. 35, 18 (1973).

[87] R. Balian and G. Toulouse, Phys. Rev. Lett. 30, 544 (1973).

[88] M. E. Fisher, Phys. Rev. Lett. 30, 679 (1973).

[89] S. Ma, Modern Theory of Critical Phenomena (W. A. Benjamin, London, 1976).

[90] P. Butera and M. Comi, preprint hep-lat /9703018 (1997).

[91] A. Z. Patashinskii and V. L. Pokrovskii, Fluctuational Theory of Phase Transitions (Moscow, Nauka, 1982).

[92] S. A. Antonenko and A. I. Sokolov, Phys. Rev. E 51, 1894 (1995).

[93] J. C. Le Guillou and J. Zinn-Justin, Phys. Rev. Lett. 39, 95 (1977).

[94] P. Ginsparg, Nucl. Phys. B 170 [FS1], 388 (1980).

[95] V. I. Yukalov and E. P. Yukalova, Nuovo Cimento 108, 1017 (1993). 


\section{Table Caption}

Critical indices, for the models with different numbers of components $n$, calculated by using self-similar exponential approximants obtained from the $\epsilon$-expansion, and compared to the results listed in literature. The indices $\nu, \eta$, and $\theta$ are calculated directly, and other indices are found from the known scaling relations. 


\begin{tabular}{|c|c|c|c|c|c|c|c|}
\hline$n$ & $\nu$ & $\eta$ & $\gamma$ & $\beta$ & $\alpha$ & $\delta$ & $\theta$ \\
\hline-2 & $1 / 2$ & 0 & 1 & $1 / 4$ & $1 / 2$ & 5 & 0.4 \\
\hline-1 & 0.545 & 0.019 & 1.08 & 0.278 & 0.365 & 4.888 & 0.431 \\
\hline 0 & $\begin{array}{c}0.589 \\
0.587 \div 0.592 \\
{[90]}\end{array}$ & $\begin{array}{c}0.03 \\
0.026 \div 0.034 \\
{[92]}\end{array}$ & $\begin{array}{c}1.16 \\
1.157 \div 1.162 \\
{[90]}\end{array}$ & $\begin{array}{c}0.303 \\
0.302 \div 0.305 \\
{[92]}\end{array}$ & $\begin{array}{c}0.233 \\
0.231 \div 0.236 \\
{[92]}\end{array}$ & 4.825 & $\begin{array}{c}0.464 \\
0.465 \pm 0.01 \\
{[91]}\end{array}$ \\
\hline 1 & $\begin{array}{c}0.632 \\
0.629 \div 0.634 \\
{[90]} \\
\end{array}$ & $\begin{array}{c}0.035 \\
0.031 \div 0.038 \\
{[92]} \\
\end{array}$ & $\begin{array}{c}1.242 \\
1.237 \div 1.244 \\
{[90]} \\
\end{array}$ & $\begin{array}{c}0.327 \\
0.324 \div 0.327 \\
{[92]} \\
\end{array}$ & $\begin{array}{c}0.104 \\
0.107 \div 0.110 \\
{[92]}\end{array}$ & 4.797 & $\begin{array}{c}0.498 \\
0.5 \pm 0.02 \\
{[91]} \\
\end{array}$ \\
\hline 2 & $\begin{array}{c}0.671 \\
0.662 \div 0.677 \\
{[90]} \\
\end{array}$ & $\begin{array}{c}0.036 \\
0.032 \div 0.039 \\
{[92]} \\
\end{array}$ & $\begin{array}{c}1.318 \\
1.308 \div 1.327 \\
{[90]} \\
\end{array}$ & $\begin{array}{c}0.348 \\
0.346 \div 0.348 \\
{[92]} \\
\end{array}$ & $\begin{array}{c}-0.013 \\
-0.007 \div-0.1 \\
{[92]}\end{array}$ & 4.792 & $\begin{array}{c}0.531 \\
0.52 \pm 0.02 \\
{[91]} \\
\end{array}$ \\
\hline 3 & $\begin{array}{c}0.708 \\
0.704 \div 0.72 \\
{[90]}\end{array}$ & $\begin{array}{c}0.037 \\
0.031 \div 0.038 \\
{[92]}\end{array}$ & $\begin{array}{c}1.39 \\
1.385 \div 1.406 \\
{[90]}\end{array}$ & $\begin{array}{c}0.367 \\
0.362 \div 0.366 \\
{[92]}\end{array}$ & $\begin{array}{c}-0.124 \\
-0.115 \div-0.117 \\
{[92]}\end{array}$ & 4.786 & $\begin{array}{c}0.562 \\
0.55 \pm 0.015 \\
{[91]}\end{array}$ \\
\hline 4 & $\begin{array}{c}0.741 \\
0.738 \div 0.755 \\
{[90]}\end{array}$ & $\begin{array}{c}0.036 \\
0.036 \\
{[92]}\end{array}$ & $\begin{array}{c}1.455 \\
1.449 \div 1.483 \\
{[90]}\end{array}$ & $\begin{array}{c}0.384 \\
0.382 \\
{[92]}\end{array}$ & $\begin{array}{c}-0.223 \\
-0.213 \\
{[92]}\end{array}$ & 4.792 & 0.592 \\
\hline 6 & $\begin{array}{c}0.797 \\
0.79 \div 0.818 \\
{[90]} \\
\end{array}$ & $\begin{array}{c}0.033 \\
0.031 \\
{[92]} \\
\end{array}$ & $\begin{array}{c}1.568 \\
1.556 \div 1.608 \\
{[90]} \\
\end{array}$ & $\begin{array}{l}0.412 \\
0.407 \\
{[92]} \\
\end{array}$ & $\begin{array}{c}-0.391 \\
-0.37 \\
{[92]}\end{array}$ & 4.808 & 0.645 \\
\hline 8 & $\begin{array}{c}0.84 \\
0.83 \div 0.856 \\
{[90]}\end{array}$ & $\begin{array}{c}0.029 \\
0.027 \\
{[92]} \\
\end{array}$ & $\begin{array}{c}1.656 \\
1.637 \div 1.687 \\
{[90]}\end{array}$ & $\begin{array}{l}0.432 \\
0.426 \\
{[92]}\end{array}$ & $\begin{array}{c}-0.52 \\
-0.489 \\
{[92]}\end{array}$ & 4.831 & 0.688 \\
\hline 10 & $\begin{array}{c}0.872 \\
0.85 \div 0.884 \\
{[90]}\end{array}$ & $\begin{array}{l}0.026 \\
0.024 \\
{[92]} \\
\end{array}$ & $\begin{array}{c}1.721 \\
1.697 \div 1.744 \\
{[90]}\end{array}$ & $\begin{array}{l}0.447 \\
0.440 \\
{[92]} \\
\end{array}$ & $\begin{array}{c}-0.616 \\
-0.576 \\
{[92]}\end{array}$ & 4.848 & 0.723 \\
\hline 12 & $\begin{array}{c}0.896 \\
0.881 \div 0.902 \\
{[90]} \\
\end{array}$ & $\begin{array}{c}0.023 \\
0.021 \\
{[92]} \\
\end{array}$ & $\begin{array}{c}1.771 \\
1.741 \div 1.783 \\
{[90]}\end{array}$ & $\begin{array}{c}0.458 \\
0.450 \\
{[92]} \\
\end{array}$ & $\begin{array}{c}-0.688 \\
-0.643 \\
{[92]}\end{array}$ & 4.865 & 0.751 \\
\hline$\infty$ & 1 & 0 & 2 & $1 / 2$ & -1 & 5 & 1 \\
\hline
\end{tabular}

